\title{
ISOMONODROMIC DEFORMATION OF FUCHSIAN PROJECTIVE CONNECTIONS ON ELLIPTIC CURVES
}

\author{
SHINGO KAWAI
}

\begin{abstract}
We consider isomonodromic deformations of second-order Fuchsian differential equations on elliptic curves. The isomonodromic deformations are described as a completely integrable Hamiltonian system.
\end{abstract}

\section{Introduction}

We consider isomonodromic deformations of second-order Fuchsian differential equations on elliptic curves. Our primary object of study is the monodromy mapping $\mathcal{F}: \mathcal{E} \rightarrow \mathcal{R}$, where $\mathcal{E}$ is the space of deformation parameters of Fuchsian equations, and $\mathcal{R}$ is the space of conjugacy classes of representations of the fundamental group of the nonsingular locus of the equations. Our goal is to describe the tangential directions to the fibers of $\mathcal{F}$ by a completely integrable system of partial differential equations on the space $\mathcal{E}$. For this purpose we use the fact that there is defined a canonical closed nondegenerate 2 -form (i.e., a symplectic structure) $\omega$ on the space $\mathcal{R}$. By pulling back this 2-form onto $\mathcal{E}$ via $\mathcal{F}$, we obtain a closed 2-form $\mathcal{F}^{*} \omega$ that describes the isomonodromic deformations as the directions making $\mathcal{F}^{*} \omega$ degenerate. As a result we find that the isomonodromic deformations are described as a completely integrable Hamiltonian system.

Our specific setting of Fuchsian equations on elliptic curves was originally treated by Okamoto [16]-[18] and then generalized by Iwasaki [6] to the case of higher genera. The main new features of our discussion here are that (i) we allow the underlying elliptic curve also vary, and that (ii) we use fully the "pulling-back" principle which was inspired by Iwasaki [7]. Isomonodromic deformations on elliptic curves have also appeared in

Received December 20, 2001.

2000 Mathematics Subject Classification: 32G34, 34M55; 32G15, 53D30.

This research was partially supported by Grant-in-Aid for Encouragement of Young Scientists (No. 12740101), the Ministry of Education, Culture, Sports, Science and Technology, Japan. 
Korotkin-Samtleben [13], Levin-Olshanetsky [14] and Takasaki [19], [20] via different methods and motivations.

This article is organized as follows: In Section 1 we introduce the linear equations, illustrate our method of studying isomonodromic deformations, and then present our main results. Section 2 contains the construction of the space $\mathcal{R}$ of representations with particular emphasis on its smooth structure and the description of its tangent spaces. In Section 3 we develop the variational theory of monodromy representations. Section 4 contains the description of the symplectic structure on $\mathcal{R}$. In view of the results of the preceding two sections, we evaluate the symplectic form on $\mathcal{R}$ in Section 5. The details of the calculation are given in Section 6 and the explicit form of the pulled-back 2-form is then obtained. Analyzing that 2-form closely, we derive the desired isomonodromy equation in Section 7. Finally in Section 8 we examine in more detail the isomonodromy equation in the simplest setting.

This article is a revised and extended version of my thesis [9] at the University of Tokyo, 1995. Part of the results here has been announced in [11].

Acknowledgements. First of all, I wish to thank Professor Mikio Furuta for introducing me to this investigation, for explaining many of the required techniques and for his constant guidance and support throughout this work. Next, I am grateful to my thesis advisor Professor Kanji Namba for encouragement and support over the years. I would also like to express my gratitude to Professor Katsuhisa Mimachi for valuable comments on earlier versions of the manuscript. Finally, I thank Professors Toshiyuki Akita, Yoshitake Hashimoto, Katsunori Iwasaki, Sadayoshi Kojima, Takayuki Oda, Kazuo Okamoto, Hidetaka Sakai, Kanehisa Takasaki and Masaaki Yoshida for interest, comments and encouragement.

\section{$\S 1$. Setting, method and results}

\subsection{Linear equations}

Let $M$ be an elliptic curve over $\mathbb{C}$, and $\mathbb{H}=\{\tau \in \mathbb{C} ; \operatorname{Im} \tau>0\}$ the upper half-plane. Selecting a suitable point $\tau \in \mathbb{H}$ realizes $M$ as the quotient $\mathbb{C} / \Gamma_{\tau}$ under the action of the lattice group $\Gamma_{\tau}$ of translations $\gamma_{l, n}^{\tau}: z \mapsto$ $z+l+n \tau,(l, n) \in \mathbb{Z}^{2}$; and then equations on $M$ are described as equations on $\mathbb{C}$ with coefficients doubly periodic with respect to $\Gamma_{\tau}$. Consider now a 
Fuchsian equation of the form

$$
\frac{d^{2} w}{d z^{2}}=q(z) w
$$

where the coefficient $q(z)$ is given by

$$
\begin{gathered}
q(z)=L+\sum_{i=0}^{m}\left[H_{i} \zeta\left(z-t_{i}, \tau\right)+\frac{1}{4}\left(\theta_{i}^{2}-1\right) \wp\left(z-t_{i}, \tau\right)\right] \\
+\sum_{\alpha=0}^{m}\left[-\mu_{\alpha} \zeta\left(z-\lambda_{\alpha}, \tau\right)+\frac{3}{4} \wp\left(z-\lambda_{\alpha}, \tau\right)\right] \\
\sum_{i=0}^{m} H_{i}-\sum_{\alpha=0}^{m} \mu_{\alpha}=0 .
\end{gathered}
$$

Here $\zeta(z, \tau)$ and $\wp(z, \tau)$ are Weierstrass' $\zeta$-function and $\wp$-function with fundamental periods $1, \tau$; and the $L, H_{i}, t_{i}, \theta_{i}, \mu_{\alpha}, \lambda_{\alpha}$ are complex parameters. In particular, we assume that the points $t_{i}, \lambda_{\alpha} \in \mathbb{C}$ are so chosen that $t_{0}=0$ and

$$
t_{i} \not \equiv t_{j}, t_{i} \not \equiv \lambda_{\alpha}, \lambda_{\alpha} \not \equiv \lambda_{\beta} \bmod \Gamma_{\tau}(i, j, \alpha, \beta=0, \ldots, m ; i \neq j, \alpha \neq \beta) .
$$

Equation (1)-(3) has its (regular) singularities at $\left[t_{i}\right](i=0, \ldots, m)$ and $\left[\lambda_{\alpha}\right](\alpha=0, \ldots, m)$ with characteristic exponents $\frac{1}{2}\left(1 \pm \theta_{i}\right)$ and $\frac{1}{2}(1 \pm$ 2 ) respectively $([z]$ denotes the congruence class of a point $z \in \mathbb{C})$ and determines its monodromy representation

$$
\rho: \pi_{1}\left(M \backslash\left\{\left[t_{0}\right], \ldots,\left[t_{m}\right],\left[\lambda_{0}\right], \ldots,\left[\lambda_{m}\right]\right\}\right) \longrightarrow S L(2, \mathbb{C}) \subset G L(2, \mathbb{C})
$$

up to conjugacy. The image group of the homomorphism $\rho$ is indeed contained in $S L(2, \mathbb{C})$ because the Wronskian of any fundamental system of solutions is independent of $z$. In particular, if we assume here that (A1) the singularities $\left[t_{i}\right]$ are generic (i.e., the parameters $\theta_{i}$ are not integers), and that (A2) the singularities $\left[\lambda_{\alpha}\right]$ are apparent (i.e., nonlogarithmic), then the local monodromy representations around the points $\left[t_{i}\right]$ and $\left[\lambda_{\alpha}\right]$ respectively become conjugate to

$$
\left[\begin{array}{cc}
-\exp \left(\pi \sqrt{-1} \theta_{i}\right) & 0 \\
0 & -\exp \left(-\pi \sqrt{-1} \theta_{i}\right)
\end{array}\right] \quad \text { and } \quad\left[\begin{array}{cc}
-1 & 0 \\
0 & -1
\end{array}\right]
$$


(For use in a later discussion, we assume further that (A3) $\sum_{i=0}^{m} \epsilon_{i} \theta_{i} \neq$ $0, \pm 2, \pm 4, \ldots$ for all $\left(\epsilon_{0}, \ldots, \epsilon_{m}\right) \in\{ \pm 1\}^{m+1}$; see Section 2.3.)

At this point we note that, as explained in Okamoto [18, pp. 288-289, 292-293], assumption (A2) and equality (3) are explicitly written as the following system of equations for the parameters $L, H_{i}$ :

$$
\left[\begin{array}{cccc}
1 & \zeta\left(\lambda_{0}-t_{0}, \tau\right) & \cdots & \zeta\left(\lambda_{0}-t_{m}, \tau\right) \\
1 & \zeta\left(\lambda_{1}-t_{0}, \tau\right) & \cdots & \zeta\left(\lambda_{1}-t_{m}, \tau\right) \\
\vdots & \vdots & \ddots & \vdots \\
1 & \zeta\left(\lambda_{m}-t_{0}, \tau\right) & \cdots & \zeta\left(\lambda_{m}-t_{m}, \tau\right) \\
0 & 1 & \cdots & 1
\end{array}\right]\left[\begin{array}{c}
L \\
H_{0} \\
\vdots \\
H_{m-1} \\
H_{m}
\end{array}\right]=\left[\begin{array}{c}
\nu_{0} \\
\nu_{1} \\
\vdots \\
\nu_{m} \\
\sum_{\alpha=0}^{m} \mu_{\alpha}
\end{array}\right],
$$

where for $\alpha=0, \ldots, m$,

$$
\begin{aligned}
\nu_{\alpha}=\mu_{\alpha}^{2}+\sum_{\beta=0, \neq \alpha}^{m}\left[\mu_{\beta} \zeta\left(\lambda_{\alpha}-\lambda_{\beta}, \tau\right)-\frac{3}{4} \wp\left(\lambda_{\alpha}-\lambda_{\beta}, \tau\right)\right] \\
-\sum_{i=0}^{m} \frac{1}{4}\left(\theta_{i}^{2}-1\right) \wp\left(\lambda_{\alpha}-t_{i}, \tau\right) .
\end{aligned}
$$

Consequently, viewing the $\theta_{i}$ as fixed non-integral constants satisfying (A3), we find that the parameters $L, H_{i}$ are then expressed as certain functions

$$
\left\{\begin{array}{l}
L=L(\tau, t, \lambda, \mu), \\
H_{i}=H_{i}(\tau, t, \lambda, \mu) \quad(i=0, \ldots, m)
\end{array}\right.
$$

of the others under the condition that the coefficient matrix of (6) be nonsingular. (We have introduced the vector notation $t=\left(t_{1}, \ldots, t_{m}\right)$, $\lambda=\left(\lambda_{0}, \ldots, \lambda_{m}\right), \mu=\left(\mu_{0}, \ldots, \mu_{m}\right)$.) According to the general theory of Fuchsian projective connections on Riemann surfaces that has been developed by Iwasaki [6, pp. 496-498, 505-507], the nonsingularity condition above is in turn guaranteed if we assume further that the $(\tau, t, \lambda, \mu)$ satisfy the condition

$$
\sum_{\alpha=0}^{m} \lambda_{\alpha}-\sum_{i=0}^{m} t_{i} \not \equiv 0 \quad \bmod \Gamma_{\tau}
$$

\subsection{Space of linear equations}

Recalling the discussion in the previous paragraph and keeping in mind that the $\theta_{i}$ are fixed non-integral constants satisfying (A3), let us introduce 
the space $\mathcal{E}$ of linear equations having the form (1)-(3) and satisfying assumptions (4), (A2), (8) as follows:

$$
\mathcal{E}=\left\{(\tau, t, \lambda, \mu) \in \mathbb{H} \times \mathbb{C}^{3 m+2} ; \text { conditions (4), (8) hold }\right\} .
$$

Since (4) and (8) are open conditions for $(\tau, t, \lambda, \mu)$, the space $\mathcal{E}$ is locally parametrized by $(\tau, t, \lambda, \mu)$ and therefore carries the structure of a complex analytic manifold of dimension $3(m+1)$. Actually, since (4) and (8) are conditions only for $(\tau, t, \lambda)$, it is more to the point to view the space $\mathcal{E}$ as a fiber space $\mathcal{E} \rightarrow \mathcal{B}$ over the set $\mathcal{B}$ of points $(\tau, t, \lambda) \in \mathbb{H} \times \mathbb{C}^{2 m+1}$ satisfying (4) and (8), or more specifically, a holomorphic affine bundle $\mathcal{E} \rightarrow \mathcal{B}$ of rank $m+1$ over $\mathcal{B}$. For details, see [6, pp. 505-507].

Let us denote by $(\tau(0), t(0), \lambda(0), \mu(0))$ some fixed point on $\mathcal{E}$, and consider a holomorphic family $(\tau(s), t(s), \lambda(s), \mu(s))$ of differential equations passing through that point, where $s$ is a complex parameter varying in the unit disk $\Delta$. (The corresponding values of the functions (7) will be denoted by $L(s)$ and $H_{i}(s)$.) We emphasize here that this is a family of differential equations on varying elliptic curves $M_{\tau(s)}=\mathbb{C} / \Gamma_{\tau(s)}$, where $\Gamma_{\tau(s)}=\left\{\gamma_{l, n}^{\tau(s)}\right.$ : $\left.z \mapsto z+l+n \tau(s) ;(l, n) \in \mathbb{Z}^{2}\right\}$, and that is exactly the main point we would like to address in this article. Our primary interest then lies in the fact that, while by construction the local monodromy representations around the points $\left[t_{i}(s)\right]$ and $\left[\lambda_{\alpha}(s)\right]$ remain constant with respect to $s$, the global monodromy of the equations $(\tau(s), t(s), \lambda(s), \mu(s))$ may in general change. If the global monodromy of $(\tau(s), t(s), \lambda(s), \mu(s))$ still remains constant (up to conjugacy), then the family will be called an isomonodromic family.

To be more precise in this respect, let us consider a sufficiently small open neighborhood $\mathcal{W}$ of the point $r_{0}=(\tau(0), t(0), \lambda(0), \mu(0))$ in $\mathcal{E}$, and denote by $r$ an arbitrary point $(\tau, t, \lambda, \mu)$ in $\mathcal{W}$. We write

$$
X_{r}=M_{\tau} \backslash\left\{\left[t_{0}\right],\left[t_{1}\right], \ldots,\left[t_{m}\right],\left[\lambda_{0}\right], \ldots,\left[\lambda_{m}\right]\right\} \quad\left(M_{\tau}=\mathbb{C} / \Gamma_{\tau}\right)
$$

and denote by $\mathcal{R}\left(X_{r_{0}}\right)=\operatorname{Hom}\left(\pi_{1}\left(X_{r_{0}}\right), S L(2, \mathbb{C})\right) / S L(2, \mathbb{C})$ the set of conjugacy classes of linear representations of $\pi_{1}\left(X_{r_{0}}\right)$ into $S L(2, \mathbb{C})$. Our starting observation is then that the monodromy of each equation $r=$ $(\tau, t, \lambda, \mu) \in \mathcal{W}$, which is a (conjugacy class of) homomorphism $\rho(r)$ : $\pi_{1}\left(X_{r}\right) \rightarrow S L(2, \mathbb{C})$, can be viewed as an element of a suitable subset $\mathcal{R}$ of $\mathcal{R}\left(X_{r_{0}}\right)$ via the canonical isomorphism $\pi_{1}\left(X_{r}\right) \cong \pi_{1}\left(X_{r_{0}}\right)$, and hence that there arises a (holomorphic) mapping

$$
\mathcal{F}: \mathcal{W} \longrightarrow \mathcal{R} \quad r \longmapsto[\rho(r)],
$$


where the set $\mathcal{R}$ carries the structure of a complex analytic manifold of dimension $2(m+1)$ (see Section 2$)$. In terms of this mapping $\mathcal{F}$, the condition for the family $(\tau(s), t(s), \lambda(s), \mu(s))$ to be isomonodromic is geometrically rephrased as the condition that there exist a fiber $\mathcal{F}^{-1}(\rho)$ of $\mathcal{F}$ containing the whole family; and our goal in this article will be to give an infinitesimal description of the tangential directions to the fibers $\mathcal{F}^{-1}(\rho)$ in terms of a completely integrable system of partial differential equations on the parameters $(\tau, t, \lambda, \mu)$ of $\mathcal{W}$.

Remark. The mapping $\mathcal{F}$, which was defined on the open neighborhood $\mathcal{W}$ of the point $r_{0}$, cannot in general be extended to the whole space $\mathcal{E}$. One way to obtain a mapping defined globally is to introduce instead of $\mathcal{B}$ (a suitable open subset of) the Teichmüller space $\mathcal{T}_{1,2 m+2}$ of elliptic curves with $2 m+2$ marked points. Another way is to construct instead of $\mathcal{R}$ another space $\mathcal{R}^{+}$of representations with a local system structure $\mathcal{R}^{+} \rightarrow \mathcal{B}$ (for details, see [6]).

\subsection{Method}

We move on to the key observation for describing the tangential directions to the fibers of $\mathcal{F}$, that is, that there exists a natural symplectic structure $\omega$ on the space $\mathcal{R}$ of representations, which was found in these settings by Iwasaki [7]. (For the symplectic structure on $\mathcal{R}$, see also BiswasGuruprasad [1] and Goldman [2].) By definition, a (complex) symplectic structure $\omega$ on $\mathcal{R}$ is a closed nondegenerate holomorphic 2-form on the complex manifold $\mathcal{R}$. By pulling back the 2 -form $\omega$ onto $\mathcal{W} \subset \mathcal{E}$ via $\mathcal{F}$, we obtain a possibly degenerate closed 2 -form on $\mathcal{W}$; and if we assume here that the differential $d_{r} \mathcal{F}$ of $\mathcal{F}$ at an arbitrary point $r \in \mathcal{W}$ has maximal rank (consequently the fibers of $\mathcal{F}$ turn out to be all smooth), then the pulled-back 2-form above can be used to describe the tangential directions to the fibers as follows: For a tangent vector $\xi$ to $\mathcal{W}$ at a point $r \in \mathcal{W}$, we have

$\xi$ is tangent to a fiber of $\mathcal{F}$

$$
\begin{aligned}
& \Longleftrightarrow d_{r} \mathcal{F}(\xi)=0 \\
& \Longleftrightarrow \omega_{\mathcal{F}(r)}\left(\cdot, d_{r} \mathcal{F}(\xi)\right) \equiv 0 \quad \text { since } \omega \text { is nondegenerate } \\
& \Longleftrightarrow\left(\mathcal{F}^{*} \omega\right)_{r}(\cdot, \xi) \equiv 0 \quad \text { since } d_{r} \mathcal{F} \text { is surjective. }
\end{aligned}
$$

It thus follows that the problem of describing the tangential directions to the fibers of $\mathcal{F}$ reduces to that of determining precisely the vectors $\xi$ 
such that $\mathcal{F}^{*} \omega(\cdot, \xi) \equiv 0$ (we say that $\mathcal{F}^{*} \omega$ is degenerate in the direction $\xi$ if this happens); and consequently our task in this article will be to write out the pulled-back 2 -form $\mathcal{F}^{*} \omega$ in terms of the local coordinates $(\tau, t, \lambda, \mu)$ and then determine the directions making $\mathcal{F}^{*} \omega$ degenerate explicitly.

\subsection{Results}

With these remarks on the basic idea of our geometric approach made, we are ready to state our main results.

THEOREM 1. In terms of the local coordinate parameters $(\tau, t, \lambda, \mu)$ on $\mathcal{W} \subset \mathcal{E}$, the pulled-back 2 -form $\mathcal{F}^{*} \omega$ takes the form

$$
\mathcal{F}^{*} \omega=-2\left(\sum_{\alpha=0}^{m} d \mu_{\alpha} \wedge d \lambda_{\alpha}-\sum_{i=1}^{m} d H_{i} \wedge d t_{i}-d K \wedge d \tau\right)
$$

where (i) the term $K$ is defined by

$$
K=\frac{1}{2 \pi \sqrt{-1}}\left[L+\eta_{1}(\tau)\left(\sum_{\alpha=0}^{m} \lambda_{\alpha} \mu_{\alpha}-\sum_{i=1}^{m} t_{i} H_{i}\right)\right]
$$

(ii) the term $\eta_{1}(\tau)$ is given by $\eta_{1}(\tau)=\zeta(z+1, \tau)-\zeta(z, \tau)$, and (iii) the $L$ and $H_{i}$ are viewed as functions of $(\tau, t, \lambda, \mu)$ that are determined by equation (6).

In particular, if we consider the space $\mathcal{E}_{0}$ of differential equations (again having the form (1)-(3) and satisfying assumptions (4), (A2) and (8)) on the fixed elliptic curve $M_{\tau(0)}=\mathbb{C} / \Gamma_{\tau(0)}$, then the resulting 2 -form $\mathcal{F}^{*} \omega$ becomes

$$
-2\left(\sum_{\alpha=0}^{m} d \mu_{\alpha} \wedge d \lambda_{\alpha}-\sum_{i=1}^{m} d H_{i} \wedge d t_{i}\right)
$$

and that formula is indeed a special instance of Iwasaki's result [7].

As we shall see in Section 7, the surjectivity condition for the differential $d_{r} \mathcal{F}$ at an arbitrary point $r \in \mathcal{W}$ follows immediately from the explicit form (9) of $\mathcal{F}^{*} \omega$. Therefore, writing out the null-distribution $\mathcal{D}=\{\xi \in T \mathcal{W}$; $\left.\mathcal{F}^{*} \omega(\cdot, \xi) \equiv 0\right\}$ of $\mathcal{F}^{*} \omega$ explicitly, we obtain the following result. 
THEOREM 2. The isomonodromic deformations of equation (1)-(3) are described by the completely integrable Hamiltonian system

$$
\left\{\begin{array}{l}
d \lambda_{\alpha}=\sum_{i=1}^{m} \frac{\partial H_{i}}{\partial \mu_{\alpha}} d t_{i}+\frac{\partial K}{\partial \mu_{\alpha}} d \tau, \quad(\alpha=0, \ldots, m) . \\
d \mu_{\alpha}=-\sum_{i=1}^{m} \frac{\partial H_{i}}{\partial \lambda_{\alpha}} d t_{i}-\frac{\partial K}{\partial \lambda_{\alpha}} d \tau
\end{array}\right.
$$

If we regard again $\tau$ as a fixed constant, then the resulting system becomes

$$
\left\{\begin{array}{l}
d \lambda_{\alpha}=\sum_{i=1}^{m} \frac{\partial H_{i}}{\partial \mu_{\alpha}} d t_{i} \\
d \mu_{\alpha}=-\sum_{i=1}^{m} \frac{\partial H_{i}}{\partial \lambda_{\alpha}} d t_{i}
\end{array} \quad(\alpha=0, \ldots, m) ;\right.
$$

and that system has been obtained by Okamoto [17], [18] and Iwasaki [6].

Now we conclude this section with our one final result, which identifies the solutions to the system (10) in the simplest case $m=0$.

THEOREM 3. For $m=0$, the completely integrable Hamiltonian system (10) reduces to the following nonlinear ordinary differential equation

$$
\frac{d^{2} \lambda}{d \tau^{2}}=-\frac{\theta^{2}}{8 \pi^{2}} \wp^{\prime}(\lambda, \tau)
$$

where we use the notation $\lambda=\lambda_{0}, \theta=\theta_{0}$ and $\wp^{\prime}(\lambda, \tau)=\frac{\partial \wp}{\partial \lambda}(\lambda, \tau)$.

According to Manin [15], this equation corresponds to a special one-parameter family of the elliptic form of the sixth Painlevé equation; and we remark that a similar result (i.e., an interpretation of Manin's formula as an isomonodromy equation on elliptic curves) for this particular case has been obtained by Levin-Olshanetsky [14] by considering a nonautonomous analogue of the two-body elliptic Calogero-Moser system. Furthermore, a similar interpretation for the full-parameter case has also been proposed by Takasaki [20]. In a forthcoming article [12], we shall address the fullparameter case again in our framework of projective connections. 


\section{$\S 2$. Spaces of representations}

In this section we construct the space $\mathcal{R}$ of representations as a suitable subset of $\mathcal{R}\left(X_{r_{0}}\right)=\operatorname{Hom}\left(\pi_{1}\left(X_{r_{0}}\right), S L(2, \mathbb{C})\right) / S L(2, \mathbb{C})$. Although most of our discussion is by now standard (see Biswas-Guruprasad [1] and Goldman [2]), our primary concern will be on the fact that some of the local representations around the deleted points are exactly $-I$. Since the set of conjugacy classes in $S L(2, \mathbb{C})$ ceases to be Hausdorff around the classes $\{I\}$ and $\{-I\}$, some special care must be taken to treat the space of such representations.

\subsection{Apparent singularities}

Now let us recall first that the fundamental group $\pi_{1}\left(X_{r_{0}}\right)$ is described as a group with $2 m+4$ generators $\left\{\alpha, \beta, \gamma_{0}, \ldots, \gamma_{m}, \delta_{0}, \ldots, \delta_{m}\right\}$ and one defining relation $\alpha \beta \alpha^{-1} \beta^{-1} \gamma_{0} \cdots \gamma_{m} \delta_{0} \cdots \delta_{m}=1$, where the $\gamma_{i}$ and $\delta_{\alpha}$ are chosen to represent respectively the (homotopy classes of) loops encircling the points $\left[t_{i}(0)\right]$ and $\left[\lambda_{\alpha}(0)\right]$ counterclockwise; and the product $\alpha \beta$ of two elements $\alpha$ and $\beta$ is represented by the loop that follows $\beta$ first and then $\alpha$. By associating to each homomorphism $\rho: \pi_{1}\left(X_{r_{0}}\right) \rightarrow$ $S L(2, \mathbb{C})$ the $2 m+4$ matrices corresponding to the generators above, the set $\operatorname{Hom}\left(\pi_{1}\left(X_{r_{0}}\right), S L(2, \mathbb{C})\right)$ is then identified with the complex analytic variety

$$
\begin{aligned}
& \mathcal{S}=\left\{\left(A, B, C_{0}, \ldots, C_{m}, D_{0}, \ldots, D_{m}\right) \in S L(2, \mathbb{C})^{2 m+4}\right. \\
&\left.A B A^{-1} B^{-1} C_{0} \cdots C_{m} D_{0} \cdots D_{m}=I\right\},
\end{aligned}
$$

which is easily seen to be smooth. Similarly, for the free group $\left\langle\delta_{\alpha}\right\rangle$ generated by the element $\delta_{\alpha}(\alpha=0, \ldots, m)$, the set $\operatorname{Hom}\left(\left\langle\delta_{\alpha}\right\rangle, S L(2, \mathbb{C})\right)$ is identified with the group $S L(2, \mathbb{C})$ itself by associating to $\rho \in \operatorname{Hom}\left(\left\langle\delta_{\alpha}\right\rangle, S L(2, \mathbb{C})\right)$ the matrix $\rho\left(\delta_{\alpha}\right)$.

Recalling that the equations $(\tau, t, \lambda, \mu) \in \mathcal{W}$ have local monodromy $-I$ around the points $\left[\lambda_{\alpha}\right]$ (see $(5)$ ), let us consider the restriction mappings $\eta_{\alpha}: \mathcal{S} \rightarrow S L(2, \mathbb{C})$ defined by $\rho \mapsto \rho\left(\delta_{\alpha}\right)$, and define a subset $\mathcal{S}_{1} \subset \mathcal{S}$ by $\mathcal{S}_{1}=\left\{\rho \in \mathcal{S} ; \eta_{\alpha}(\rho)=-I(\alpha=0, \ldots, m)\right\}$, which is also seen to be smooth. A standard argument (refer to [2, p. 203] and Gunning [4, pp. 184-185], [5, p. 51]) shows that

(i) the differential of the mapping $\eta_{\alpha}$ at a point $\rho$ is naturally identified with the homomorphism

$$
\iota_{\alpha}(\rho): Z^{1}\left(\pi_{1}\left(X_{r_{0}}\right), \mathfrak{g}_{\mathrm{Ad} \rho}\right) \longrightarrow Z^{1}\left(\left\langle\delta_{\alpha}\right\rangle, \mathfrak{g}_{\mathrm{Ad} \rho} \mid\left\langle\delta_{\alpha}\right\rangle\right),
$$


where $Z^{1}\left(\pi_{1}\left(X_{r_{0}}\right), \mathfrak{g}_{\text {Ad } \rho}\right)$ denotes the space of cocycles of the group $\pi_{1}\left(X_{r_{0}}\right)$ with coefficients in the $\pi_{1}\left(X_{r_{0}}\right)$-module $\mathfrak{g}=\mathfrak{s l}(2, \mathbb{C})$ under the group representation $\operatorname{Ad} \rho$, and similarly for $Z^{1}\left(\left\langle\delta_{\alpha}\right\rangle, \mathfrak{g}_{\operatorname{Ad} \rho \mid\left\langle\delta_{\alpha}\right\rangle}\right)$.

It thus follows from the definition that the tangent space $T_{\rho} \mathcal{S}_{1}$ to the submanifold $\mathcal{S}_{1} \subset \mathcal{S}$ at a point $\rho \in \mathcal{S}_{1}$ is identified with the intersection of the kernels of the mappings $\iota_{\alpha}(\rho)(\alpha=0, \ldots, m)$ :

$$
T_{\rho} \mathcal{S}_{1}=\bigcap_{\alpha=0}^{m} \operatorname{Ker} \iota_{\alpha}(\rho) .
$$

\subsection{Action by inner automorphisms}

Let us consider next the action of $S L(2, \mathbb{C})$, or more appropriately of $P S L(2, \mathbb{C})=S L(2, \mathbb{C}) /\{ \pm I\}$, on the manifold $\mathcal{S}_{1}$ by inner automorphisms. To obtain an action with a reasonable quotient space, we restrict attention to the invariant open subset $\mathcal{S}_{1}^{-} \subset \mathcal{S}_{1}$ consisting of those representations having only scalar commutants (compare with [2, pp. 204-205] and [5, pp. 51-53]). Exactly as in [5, Thm. 2] (see also [4, pp. 190-197]), we then find that

(ii) the quotient space $\mathcal{R}_{1}=\mathcal{S}_{1}^{-} / P S L(2, \mathbb{C})$ has the structure of a complex analytic manifold of dimension $3(m+1)$, such that the natural projection $\mathcal{S}_{1}^{-} \rightarrow \mathcal{R}_{1}$ is a complex analytic principal $\operatorname{PSL}(2, \mathbb{C})$ bundle,

(iii) the tangent space $T_{[\rho]} \mathcal{R}_{1}$ to the manifold $\mathcal{R}_{1}$ at a point $[\rho]$ corresponding to $\rho \in \mathcal{S}_{1}^{-}$is identified with the image space of $T_{\rho} \mathcal{S}_{1}^{-} \subset$ $Z^{1}\left(\pi_{1}\left(X_{r_{0}}\right), \mathfrak{g}_{\text {Ad } \rho}\right)$ under the natural mapping $Z^{1}\left(\pi_{1}\left(X_{r_{0}}\right), \mathfrak{g}_{\text {Ad } \rho}\right) \rightarrow$ $H^{1}\left(\pi_{1}\left(X_{r_{0}}\right), \mathfrak{g}_{\mathrm{Ad} \rho}\right)$, where

$$
H^{1}\left(\pi_{1}\left(X_{r_{0}}\right), \mathfrak{g}_{\operatorname{Ad} \rho}\right)=Z^{1}\left(\pi_{1}\left(X_{r_{0}}\right), \mathfrak{g}_{\operatorname{Ad} \rho}\right) / B^{1}\left(\pi_{1}\left(X_{r_{0}}\right), \mathfrak{g}_{\operatorname{Ad} \rho}\right) .
$$

Consequently it follows from (11) that $T_{[\rho]} \mathcal{R}_{1}$ can be viewed as a vector subspace of the intersection of the kernels of the natural mappings $\bar{\iota}_{\alpha}(\rho)$ : $H^{1}\left(\pi_{1}\left(X_{r_{0}}\right), \mathfrak{g}_{\mathrm{Ad} \rho}\right) \rightarrow H^{1}\left(\left\langle\delta_{\alpha}\right\rangle, \mathfrak{g}_{\mathrm{Ad} \rho} \rho\left\langle\delta_{\alpha}\right\rangle\right)$ that are induced from $\iota_{\alpha}(\rho)$. Furthermore, since it follows easily from the definition of one-coboundaries ([4, p. 128]) that $B^{1}\left(\left\langle\delta_{\alpha}\right\rangle, \mathfrak{g}_{\mathrm{Ad} \rho} \mid\left\langle\delta_{\alpha}\right\rangle\right)=0$ for $\rho \in \mathcal{S}_{1}^{-}$, we conclude that $T_{[\rho]} \mathcal{R}_{1}$ coincides precisely with the intersection above:

$$
T_{[\rho]} \mathcal{R}_{1}=\bigcap_{\alpha=0}^{m} \operatorname{Ker} \bar{\iota}_{\alpha}(\rho)
$$




\subsection{Generic singularities}

Finally we take local representations around the points $\left[t_{i}(0)\right]$ into consideration, and define the desired space $\mathcal{R}$ as a suitable subset of $\mathcal{R}_{1}$. As before, identifying the set $\operatorname{Hom}\left(\left\langle\gamma_{i}\right\rangle, S L(2, \mathbb{C})\right)$ with the group $S L(2, \mathbb{C})$ itself, let us introduce the restriction mappings $p_{i}: \mathcal{R}_{1} \rightarrow \mathfrak{C}(i=0, \ldots, m)$ defined by $[\rho] \mapsto\left[\rho\left(\gamma_{i}\right)\right]$, where $\mathfrak{C}$ denotes the set of conjugacy classes in $S L(2, \mathbb{C})$, and $[\cdot]$ indicates taking equivalence classes. Other than the sets $\{I\}$ and $\{-I\}$, conjugacy classes in $S L(2, \mathbb{C})$ are determined by the trace function $\operatorname{tr}: S L(2, \mathbb{C}) \rightarrow \mathbb{C}$; they are of the form $\operatorname{tr}^{-1}(c)$ for some $c \in \mathbb{C} \backslash\{ \pm 2\}$, or of the form $\operatorname{tr}^{-1}(2) \backslash\{I\}$ or $\operatorname{tr}^{-1}(-2) \backslash\{-I\}$. Moreover a standard argument again shows that

(iv) the subset $\mathfrak{C}^{-}=\mathfrak{C} \backslash\{\{I\},\{-I\}\}$ of $\mathfrak{C}$ has the structure of a complex analytic manifold of dimension 1 , and explicitly it is identified with $\mathbb{C}$ under the trace function (compare with Iwasaki $[7$, p. 328]),

(v) the tangent space to the manifold $\mathfrak{C}^{-}$at a point $[\rho]$ corresponding to $\rho \in S L(2, \mathbb{C}) \backslash\{ \pm I\}$ is identified with the cohomology group $H^{1}\left(\left\langle\gamma_{i}\right\rangle, \mathfrak{g}_{\mathrm{Ad} \rho}\right)$.

Now, recalling that the local monodromy of the equations $(\tau, t, \lambda, \mu) \in$ $\mathcal{W}$ around the points $\left[t_{i}\right]$ are as in (5) and introducing the mapping $p$ : $\mathcal{R}_{1} \rightarrow \mathfrak{C}^{m+1}$ of the form $[\rho] \mapsto\left(p_{0}([\rho]), \ldots, p_{m}([\rho])\right)$, we define the space $\mathcal{R}$ by

$$
\mathcal{R}=\left\{[\rho] \in \mathcal{R}_{1} ; p([\rho])=\left(-2 \cos \pi \theta_{0}, \ldots,-2 \cos \pi \theta_{m}\right)\right\},
$$

where $\mathfrak{C}^{-} \subset \mathfrak{C}$ is identified with $\mathbb{C}$ as above. (Since the $\theta_{i}$ are non-integral by assumption (A1), we have $-2 \cos \pi \theta_{i} \neq \pm 2$.) Under the additional assumption that (A3) $\sum_{i=0}^{m} \epsilon_{i} \theta_{i} \neq 0, \pm 2, \pm 4, \ldots$ for all $\left(\epsilon_{0}, \ldots, \epsilon_{m}\right) \in\{ \pm 1\}^{m+1}$, we can prove that

(vi) the differential of the mapping $p$ at any point $[\rho] \in \mathcal{R}$ corresponding to $\rho \in \mathcal{S}_{1}^{-}$, which coincides with the restriction to $T_{[\rho]} \mathcal{R}_{1}$ of the natural homomorphism $\pi(\rho): H^{1}\left(\pi_{1}\left(X_{r_{0}}\right), \mathfrak{g}_{\operatorname{Ad} \rho}\right) \rightarrow \bigoplus_{i=0}^{m} H^{1}\left(\left\langle\gamma_{i}\right\rangle, \mathfrak{g}_{\operatorname{Ad} \rho} \mid\left\langle\gamma_{i}\right\rangle\right)$, is surjective,

(vii) therefore the space $\mathcal{R}$ thus defined has the structure of a complex analytic manifold of dimension $2(m+1)$,

(viii) the tangent space $T_{[\rho]} \mathcal{R}$ to the manifold $\mathcal{R}$ at a point $[\rho]$ is identified with the intersection of the kernels of the mappings $\pi_{i}(\rho)$ : $H^{1}\left(\pi_{1}\left(X_{r_{0}}\right), \mathfrak{g}_{\mathrm{Ad} \rho}\right) \rightarrow H^{1}\left(\left\langle\gamma_{i}\right\rangle, \mathfrak{g}_{\mathrm{Ad} \rho} \rho\left\langle\gamma_{i}\right\rangle\right)$ and $\bar{\iota}_{\alpha}(\rho)$ (see $\left.(12)\right)$ : 


$$
T_{[\rho]} \mathcal{R}=\left(\bigcap_{i=0}^{m} \operatorname{Ker} \pi_{i}(\rho)\right) \cap\left(\bigcap_{\alpha=0}^{m} \operatorname{Ker} \bar{\iota}_{\alpha}(\rho)\right),
$$

and these are the main observations necessary to our discussion in later sections.

Remarks. Assertion (vi) above is verified by an elementary argument using the following items:

(a) for any subgroup $G \subset S L(2, \mathbb{C}), G$ has only scalar commutants (in $\mathfrak{g l}(2, \mathbb{C}))$ if and only if $G$ is non-abelian;

(b) the differential of the mapping $\varphi: S L(2, \mathbb{C})^{2} \rightarrow S L(2, \mathbb{C})$ defined by $(A, B) \mapsto A B A^{-1} B^{-1}$ is surjective at a point $(A, B)$ precisely when $A$ and $B$ do not commute;

(c) the differential of the mapping $\psi: S L(2, \mathbb{C})^{m} \rightarrow \mathbb{C}^{m+1}$ defined by

$$
\left(C_{1}, \ldots, C_{m}\right) \longmapsto\left(\operatorname{tr} C_{1}, \ldots, \operatorname{tr} C_{m}, \operatorname{tr}\left(C_{1} \cdots C_{m}\right)\right)
$$

is surjective at a point $\left(C_{1}, \ldots, C_{m}\right)$ precisely when $C_{1}, \ldots, C_{m}$, $C_{1} \cdots C_{m} \neq \pm I$ and $C_{1}, \ldots, C_{m}$ do not commute.

\subsection{Holomorphic curve}

In the course of the proof of assertion (vi) above, we find in particular that under assumption (A3), any representation $\rho: \pi_{1}\left(X_{r_{0}}\right) \rightarrow S L(2, \mathbb{C})$ satisfying $\operatorname{tr} \rho\left(\gamma_{i}\right)=-2 \cos \pi \theta_{i}, \rho\left(\delta_{\alpha}\right)=-I(i, \alpha=0, \ldots, m)$ necessarily becomes non-abelian, hence has only scalar commutants by item (a). Consequently the monodromy $\rho(r): \pi_{1}\left(X_{r}\right) \rightarrow S L(2, \mathbb{C})$ of each equation $r=(\tau, t, \lambda, \mu) \in \mathcal{W}$ indeed determines an element of the set $\mathcal{R}$ via the canonical isomorphism $\pi_{1}\left(X_{r}\right) \cong \pi_{1}\left(X_{r_{0}}\right)$; and thus there arises in particular from the family $(\tau(s), t(s), \lambda(s), \mu(s))$ of equations ( $s$ varies in the unit disk $\Delta$; see Section 1.2) a holomorphic curve

$$
\mathcal{C}: \Delta \longrightarrow \mathcal{R} \quad s \longmapsto[\rho(s)]
$$

in the manifold $\mathcal{R}$, where $[\rho(s)]$ denotes the monodromy of $(\tau(s), t(s), \lambda(s)$, $\mu(s))$. Our next goal will be to describe the tangent vector to this curve at the point $[\rho(0)]$ in terms of the twisted de Rham cohomology for flat connections. 


\section{$\S 3 . \quad$ Variations of monodromy representations}

Let us turn next to describing the infinitesimal variation of the monodromy representations of the equations $(\tau(s), t(s), \lambda(s), \mu(s))$ at $s=0$, or in more geometric terms, the tangent vector to the holomorphic curve (14) at the point $[\rho(0)]$. For this purpose we first reformulate the family in the language of vector bundles and connections; compare with Gunning [3, pp. 192-201], Iwasaki [6] and [10, pp. 171-175]. In what follows we shall use the notation $\Gamma_{s}=\left\{\gamma_{l, n}^{\tau(s)}: z \mapsto z+l+n \tau(s) ;(l, n) \in \mathbb{Z}^{2}\right\}, M_{s}=\mathbb{C} / \Gamma_{s}$ and $X_{s}=M_{s} \backslash\left\{\left[t_{0}\right],\left[t_{1}(s)\right], \ldots,\left[t_{m}(s)\right],\left[\lambda_{0}(s)\right], \ldots,\left[\lambda_{m}(s)\right]\right\}$.

\subsection{Bundles and connections}

Let $E_{s}$ be a trivial holomorphic vector bundle of rank 2 over $M_{s}$ and fix a trivialization $E_{s}=M_{s} \times \mathbb{C}^{2}$ throughout. Via the representation $M_{s}=\mathbb{C} / \Gamma_{s}$ above, a meromorphic connection $\nabla_{s}: \mathcal{M}\left(E_{s}\right) \rightarrow \mathcal{M}\left(E_{s} \otimes K_{s}\right)$ on $E_{s}$ is then described as $\nabla_{s}=d+\Omega_{s}$, where $\Omega_{s}$ is a $2 \times 2$ matrix of meromorphic differential forms of type $(1,0)$ on $\mathbb{C}$ that are invariant with respect to $\Gamma_{s}$. (Here $\mathcal{M}\left(E_{s}\right)$ and $\mathcal{M}\left(E_{s} \otimes K_{s}\right)$ are the sheaf of germs of meromorphic crosssections of $E_{s}$ and $E_{s} \otimes K_{s}$ respectively ( $K_{s}$ the canonical line bundle on $\left.M_{s}\right)$.) From the differential equation

$$
\frac{d^{2} w}{d z^{2}}=q_{s}(z) w
$$

with $q_{s}(z)$ given by substituting $(\tau, t, \lambda, \mu)=(\tau(s), t(s), \lambda(s), \mu(s)), L=$ $L(s)$ and $H_{i}=H_{i}(s)$ into $q(z)$ (see (1)-(3)), there naturally arises a meromorphic connection $\nabla_{s}=d+\Omega_{s}$ on $E_{s}$ by

$$
\Omega_{s}=A_{s}(z) d z=\left(\begin{array}{cc}
0 & -1 \\
-q_{s}(z) & 0
\end{array}\right) d z
$$

and the monodromy representation $\rho\left(\nabla_{s}\right): \pi_{1}\left(X_{s}\right) \rightarrow S L(2, \mathbb{C})$ of $\nabla_{s}$ is clearly the same as that of equation (15). See [3, pp. 197-201] and [6, pp. 447-449, 468-469].

We are now ready to describe the infinitesimal variation of the monodromy representations of the connections $\nabla_{s}$ at $s=0$. (A similar argument can be found in [10, pp. 173-175].) Since the mapping $\mathcal{C}$ in (14) is defined via the canonical isomorphisms $\pi_{1}\left(X_{s}\right) \cong \pi_{1}\left(X_{0}\right)$ for $s$ near 0 , we first need to pull back the connection $\nabla_{s}$ on $E_{s}$ to a connection on $E_{0}$ by some $C^{\infty}$ isomorphism $\Phi_{s}: E_{0} \rightarrow E_{s}$ that covers an (orientation-preserving) diffeomorphism $\varphi_{s}: M_{0} \rightarrow M_{s}$. (The $\Phi_{s}$ and $\varphi_{s}$ are required to depend 
holomorphically on $s$ and to satisfy $\Phi_{0}=\mathrm{id}_{E_{0}}$ and $\varphi_{0}=\mathrm{id}_{M_{0}}$.) In terms of the lattice groups $\Gamma_{0}$ and $\Gamma_{s}$, such a diffeomorphism $\varphi_{s}: M_{0} \rightarrow M_{s}$ is described by a diffeomorphism $u_{s}: \mathbb{C} \rightarrow \mathbb{C}$ that conjugates $\Gamma_{0}$ into $\Gamma_{s}$ so that the generators $\gamma_{1,0}^{\tau(0)}, \gamma_{0,1}^{\tau(0)}$ correspond to $\gamma_{1,0}^{\tau(s)}, \gamma_{0,1}^{\tau(s)}$ respectively, or more explicitly,

$$
\left\{\begin{array}{l}
u_{s}(z+1)=u_{s}(z)+1 \\
u_{s}(z+\tau(0))=u_{s}(z)+\tau(s)
\end{array}\right.
$$

for all $z \in \mathbb{C}$. To avoid getting side-tracked, an explicit construction of such a $u_{s}$ will be given in Section 5. Then, letting $\Phi_{s}$ be simply the identity in the fiber directions, the meromorphic connection $\nabla_{s}$ pulls back to a $C^{\infty}$ flat connection $\nabla_{s}^{*}=d+\Omega_{s}^{*}$ on $E_{0} \mid\left(M_{0} \backslash\left(\varphi_{s}\right)^{-1}\left(D_{s}\right)\right)$ that is given by

$$
\Omega_{s}^{*}=u_{s}^{*} \Omega_{s},
$$

where $D_{s}=\left\{\left[t_{0}\right],\left[t_{1}(s)\right], \ldots,\left[t_{m}(s)\right],\left[\lambda_{0}(s)\right], \ldots,\left[\lambda_{m}(s)\right]\right\}$ denotes the set of singular points of the connection $\nabla_{s}$; and thus we obtain a holomorphic family of singular $C^{\infty}$ flat connections $\nabla_{s}^{*}$ on the fixed bundle $E_{0}$.

\subsection{Twisted de Rham cohomology}

The main step for the description of the desired infinitesimal variation is to differentiate the equality $\Omega_{s}^{*}=\gamma^{*} \Omega_{s}^{*}$ (for each $\gamma \in \Gamma_{0}$ ) with respect to $s$ and set $s=0$. Before explaining the meaning of the resulting formula $\dot{\Omega}_{0}^{*}=\gamma^{*} \dot{\Omega}_{0}^{*}$ (the differentiation with respect to $s$ will be denoted by dots), remarks are in order. First, writing the matrix $\Omega_{s}$ as in (16), we can calculate the term $\dot{\Omega}_{0}^{*}$ as follows. Differentiating the defining formula $\Omega_{s}^{*}=$ $u_{s}^{*} \Omega_{s}=A_{s}\left(u_{s}(z)\right) d\left(u_{s}(z)\right)$ with respect to $s$ and setting $s=0$ gives

$$
\begin{aligned}
\dot{\Omega}_{0}^{*} & =\left[\dot{A}_{0}\left(u_{0}(z)\right)+A_{0}^{\prime}\left(u_{0}(z)\right) \dot{u}_{0}(z)\right] d\left(u_{0}(z)\right)+A_{0}\left(u_{0}(z)\right) d\left(\dot{u}_{0}(z)\right) \\
& =\left(\dot{A}_{0}+A_{0}^{\prime} \dot{u}_{0}\right) d z+A_{0} d \dot{u}_{0}
\end{aligned}
$$

where we have used $u_{0}(z)=z$. (The differentiation with respect to $z$ will be denoted by primes.) Second, to the vector bundle $E_{0}=M_{0} \times \mathbb{C}^{2}$, there is canonically associated another vector bundle $\operatorname{Ad} E_{0}=M_{0} \times \mathfrak{s l}(2, \mathbb{C})$. The meromorphic connection $\nabla_{0}$ on $E_{0}$ induces a connection $\hat{\nabla}_{0}$ on $\operatorname{Ad} E_{0}$ that is described as $\hat{\nabla}_{0}=d+\left[\Omega_{0}, \cdot\right]$, where $[A, B]=A B-B A$. As a $C^{\infty}$ flat connection, $\hat{\nabla}_{0}$ is defined on the restriction $\operatorname{Ad} E_{0}^{-}$of $\operatorname{Ad} E_{0}$ to the open subset $X_{0}=M_{0} \backslash D_{0}$ and determines the flat sheaf $\mathcal{F}\left(\operatorname{Ad} E_{0}^{-}\right)$of germs of horizontal cross-sections of $\operatorname{Ad} E_{0}^{-}$. 
Let $\mathcal{E}^{p}\left(\operatorname{Ad} E_{0}^{-}\right)(p=0,1,2)$ be the sheaf of germs of $C^{\infty} p$-forms with coefficients in the bundle $\operatorname{Ad} E_{0}^{-}$. The connection $\widehat{\nabla}_{0}$ then induces the covariant exterior differentiations $\widehat{\nabla}_{0}: \mathcal{E}^{p}\left(\operatorname{Ad} E_{0}^{-}\right) \rightarrow \mathcal{E}^{p+1}\left(\operatorname{Ad} E_{0}^{-}\right)(p=$ $0,1,2)$; and from the flatness of $\widehat{\nabla}_{0}$ there arises an exact sequence of sheaves

$$
0 \longrightarrow \mathcal{F}\left(\operatorname{Ad} E_{0}^{-}\right) \longrightarrow \mathcal{E}^{0}\left(\operatorname{Ad} E_{0}^{-}\right) \stackrel{\hat{\nabla}_{0}}{\longrightarrow} \mathcal{E}^{1}\left(\operatorname{Ad} E_{0}^{-}\right) \stackrel{\hat{\nabla}_{0}}{\longrightarrow} \mathcal{E}^{2}\left(\operatorname{Ad} E_{0}^{-}\right) \longrightarrow 0 .
$$

Now, since the sheaves $\mathcal{E}^{p}\left(\operatorname{Ad} E_{0}^{-}\right)$are fine, we obtain from this exact sequence the (twisted) de Rham isomorphisms

$$
\begin{aligned}
& H^{1}\left(X_{0}, \mathcal{F}\left(\operatorname{Ad} E_{0}^{-}\right)\right) \cong \frac{\operatorname{Ker} \widehat{\nabla}_{0}: \Gamma\left(X_{0}, \mathcal{E}^{1}\left(\operatorname{Ad} E_{0}^{-}\right)\right) \rightarrow \Gamma\left(X_{0}, \mathcal{E}^{2}\left(\operatorname{Ad} E_{0}^{-}\right)\right)}{\operatorname{Im} \widehat{\nabla}_{0}: \Gamma\left(X_{0}, \mathcal{E}^{0}\left(\operatorname{Ad} E_{0}^{-}\right)\right) \rightarrow \Gamma\left(X_{0}, \mathcal{E}^{1}\left(\operatorname{Ad} E_{0}^{-}\right)\right)} \\
& H_{c}^{1}\left(X_{0}, \mathcal{F}\left(\operatorname{Ad} E_{0}^{-}\right)\right) \cong \frac{\operatorname{Ker} \widehat{\nabla}_{0}: \Gamma_{c}\left(X_{0}, \mathcal{E}^{1}\left(\operatorname{Ad} E_{0}^{-}\right)\right) \rightarrow \Gamma_{c}\left(X_{0}, \mathcal{E}^{2}\left(\operatorname{Ad} E_{0}^{-}\right)\right)}{\operatorname{Im} \widehat{\nabla}_{0}: \Gamma_{c}\left(X_{0}, \mathcal{E}^{0}\left(\operatorname{Ad} E_{0}^{-}\right)\right) \rightarrow \Gamma_{c}\left(X_{0}, \mathcal{E}^{1}\left(\operatorname{Ad} E_{0}^{-}\right)\right)}
\end{aligned}
$$

where $\Gamma_{c}\left(X_{0}, \mathcal{E}^{p}\left(\operatorname{Ad} E_{0}^{-}\right)\right)$denotes the space of $C^{\infty} p$-forms with compact support and with coefficients in the bundle $\operatorname{Ad} E_{0}^{-}$. These isomorphisms will be used throughout the discussion below. For an open subset $U$ of $X_{0}$ with inclusion $j: U \rightarrow X_{0}$, we shall write simply $\mathcal{F}\left(\operatorname{Ad} E_{0}^{-}\right)$instead of $\mathcal{F}\left(\operatorname{Ad} E_{0}^{-}\right) \mid U$. The inclusion $j$ then gives rise to the natural homomorphisms

$$
\begin{array}{r}
j^{*}: H^{1}\left(X_{0}, \mathcal{F}\left(\operatorname{Ad} E_{0}^{-}\right)\right) \longrightarrow H^{1}\left(U, \mathcal{F}\left(\operatorname{Ad} E_{0}^{-}\right)\right), \\
j !: H_{c}^{1}\left(U, \mathcal{F}\left(\operatorname{Ad} E_{0}^{-}\right)\right) \longrightarrow H_{c}^{1}\left(X_{0}, \mathcal{F}\left(\operatorname{Ad} E_{0}^{-}\right)\right) ;
\end{array}
$$

in terms of differential forms, the first is expressed by restricting closed forms on $X_{0}$ to the subset $U$, and the second by extending closed forms on $U$ (with compact support) to those on $X_{0}$ by zero.

\subsection{Infinitesimal variations}

Returning to the formula $\dot{\Omega}_{0}^{*}=\gamma^{*} \dot{\Omega}_{0}^{*}$ above, we note from (16) that the matrix $\dot{\Omega}_{0}^{*}$ can be viewed as defining a global $C^{\infty} 1$-form on $X_{0}$ with coefficients in the bundle $\operatorname{Ad} E_{0}^{-}$, that is, $\dot{\Omega}_{0}^{*} \in \Gamma\left(X_{0}, \mathcal{E}^{1}\left(\operatorname{Ad} E_{0}^{-}\right)\right)$. To see that this 1 -form is closed, we consider on the complex plane $\mathbb{C}$ the following two systems of linear differential equations with parameter $s$ :

$$
\begin{aligned}
& d y+\Omega_{s} y=0, \\
& d y+\Omega_{s}^{*} y=0 .
\end{aligned}
$$

(Note that, whereas (21) is a system of ordinary differential equations with meromorphic coefficients, (22) is a system with $C^{\infty}$ singular coefficients.) 
Let $Y(z, s)$ be a fundamental solution of (21), which is by definition a holomorphic nonsingular matrix solution of (21) at some point $\left(z_{0}, s_{0}\right)$ such that $\left[z_{0}\right] \notin D_{s_{0}}$ (see Iwasaki et al. [8, p. 155]). From the definition of the pulled-back connection $\nabla_{s}^{*}$ (see $(18)$ ), it then follows that the matrix

$$
Y^{*}(z, s)=Y\left(u_{s}(z), s\right)
$$

in turn serves as a nonsingular matrix solution of (22) that is holomorphic with respect to $s$; and the closedness of the 1 -form $\dot{\Omega}_{0}^{*}$ now follows from the following simple observation.

LEMMA 1. When viewed as a local $C^{\infty}$ cross-section of the bundle Ad $E_{0}^{-}$, the matrix $\dot{Y}^{*}(z, 0) Y^{*}(z, 0)^{-1}$ satisfies

$$
\widehat{\nabla}_{0}\left(\dot{Y}^{*}(z, 0) Y^{*}(z, 0)^{-1}\right)=-\dot{\Omega}_{0}^{*}
$$

where $\widehat{\nabla}_{0}: \mathcal{E}^{0}\left(\operatorname{Ad} E_{0}^{-}\right) \rightarrow \mathcal{E}^{1}\left(\operatorname{Ad} E_{0}^{-}\right)$denotes the covariant exterior differentiation.

Proof. This is a simple calculation using $\Omega_{0}^{*}=\Omega_{0}$. It should be noted from (23) that the matrix $\dot{Y}^{*}(z, 0) Y^{*}(z, 0)^{-1}$ can indeed be assumed to have trace zero. For this we recall that the Wronskian of any fundamental solution $Y(z, s)$ of $(21)$ is constant with respect to $z$ and that, by setting a suitable initial condition, it can also be assumed to be constant with respect to $s$ as well. See also [8, pp. 154-158].

In particular, the local behavior of the 1 -form $\dot{\Omega}_{0}^{*}$ around the deleted points $\left[t_{i}(0)\right]$ and $\left[\lambda_{\alpha}(0)\right] \in M_{0}$ is seen in the following way. For convenience let us write

$$
\begin{aligned}
& a_{0}(s)=t_{0}, a_{1}(s)=t_{1}(s), \ldots, a_{m}(s)=t_{m}(s), \\
& a_{m+1}(s)=\lambda_{0}(s), \ldots, a_{2 m+1}(s)=\lambda_{m}(s) .
\end{aligned}
$$

In view of (16), assumptions (A1) and (A2) (in Section 1.1) imply that the system $(21)$ admits local fundamental solutions $Y_{k}(z, s)(k=0, \ldots, 2 m+1)$ of the form

$$
Y_{k}(z, s)=P_{k}(z, s) \exp \left[\Lambda_{k} \log \left(z-a_{k}(s)\right)\right]
$$

where (i) $P_{k}(z, s)$ is a nonsingular matrix of meromorphic functions in $z$ having at most a pole at $z=a_{k}(s)$ and depending holomorphically on 
$s$, and (ii) $\Lambda_{k}$ is a constant diagonal matrix independent of $s$. An easy calculation then shows that

$$
\dot{Y}_{k}(z, 0) Y_{k}(z, 0)^{-1}=\dot{P}_{k}(z, 0) P_{k}(z, 0)^{-1}+P_{k}(z, 0) \Lambda_{k} P_{k}(z, 0)^{-1} \frac{-\dot{a}_{k}(0)}{z-a_{k}(0)},
$$

hence that, while the matrix $Y_{k}(z, s)$ is multivalued around $z=a_{k}(s)$, the resulting matrix $\dot{Y}_{k}(z, 0) Y_{k}(z, 0)^{-1}$ becomes single-valued around $z=a_{k}(0)$. Furthermore the term $Y_{k}^{*}(z, s)=Y_{k}\left(u_{s}(z), s\right)$ in turn becomes a matrix solution of (22) as before, and we readily calculate

$$
\dot{Y}_{k}^{*}(z, 0) Y_{k}^{*}(z, 0)^{-1}=\dot{Y}_{k}(z, 0) Y_{k}(z, 0)^{-1}+Y_{k}^{\prime}(z, 0) Y_{k}(z, 0)^{-1} \dot{u}_{0}(z) .
$$

We thus find from $(26)$, (27) that the matrix $\dot{Y}_{k}^{*}(z, 0) Y_{k}^{*}(z, 0)^{-1}$ also becomes single-valued around $z=a_{k}(0)$ and hence from Lemma 1 that the 1-form $\dot{\Omega}_{0}^{*}$ above becomes exact when restricted to a sufficiently small punctured disk around each $\left[a_{k}(0)\right]$.

To be more precise, let us take sufficiently small open disks $U_{k}(k=$ $0, \ldots, 2 m+1)($ in $\mathbb{C})$ centered at each point $a_{k}(0)$ and let $U_{k}^{\prime}=U_{k} \backslash\left\{a_{k}(0)\right\}$. (In what follows we shall consider $U_{k}$ and $U_{k}^{\prime}$ also as disjoint open subsets of $M_{0}$ and $X_{0}=M_{0} \backslash D_{0}$ respectively.) It then follows from the argument so far that via the first isomorphism in $(20)$ the 1 -form $\left(-\dot{\Omega}_{0}^{*}\right)$ represents a cohomology class $\left[\left(-\dot{\Omega}_{0}^{*}\right)\right] \in H^{1}\left(X_{0}, \mathcal{F}\left(\operatorname{Ad} E_{0}^{-}\right)\right)$that is in the intersection of the kernels of the homomorphisms

$$
j_{k}^{*}: H^{1}\left(X_{0}, \mathcal{F}\left(\operatorname{Ad} E_{0}^{-}\right)\right) \longrightarrow H^{1}\left(U_{k}^{\prime}, \mathcal{F}\left(\operatorname{Ad} E_{0}^{-}\right)\right) \quad(k=0, \ldots, 2 m+1),
$$

where $j_{k}: U_{k}^{\prime} \rightarrow X_{0}$ denotes the inclusion; and this cohomology class just represents the tangent vector to the holomorphic curve (14) at the point $[\rho(0)]$ (for details, see [4, Thm. 31]). Indeed it should be noted here from [4, Thm. 19] that, with the same notation as before (Section 2), there are natural isomorphisms

$$
\begin{aligned}
& H^{1}\left(X_{0}, \mathcal{F}\left(\operatorname{Ad} E_{0}^{-}\right)\right) \cong H^{1}\left(\pi_{1}\left(X_{0}\right), \mathfrak{g}_{\operatorname{Ad} \rho}\right), \\
& H^{1}\left(U_{k}^{\prime}, \mathcal{F}\left(\operatorname{Ad} E_{0}^{-}\right)\right) \cong H^{1}\left(\left\langle\gamma_{i}\right\rangle, \mathfrak{g}_{\operatorname{Ad} \rho \mid\left\langle\gamma_{i}\right\rangle}\right) \text { or } H^{1}\left(\left\langle\delta_{\alpha}\right\rangle, \mathfrak{g}_{\operatorname{Ad} \rho \mid\left\langle\delta_{\alpha}\right\rangle}\right)
\end{aligned}
$$

(we set $\rho=\rho(0)$ ), and under these identifications the homomorphisms $j_{k}^{*}$ above coincide with the mappings $\pi_{i}(\rho)$ and $\bar{\iota}_{\alpha}(\rho)$ respectively. Consequently, in view of (13), we have verified that the cohomology class $\left[\left(-\dot{\Omega}_{0}^{*}\right)\right] \in H^{1}\left(X_{0}, \mathcal{F}\left(\operatorname{Ad} E_{0}^{-}\right)\right)$indeed determines an element of the tangent space $T_{[\rho]} \mathcal{R}$ to the manifold $\mathcal{R}$ at $[\rho]=[\rho(0)]$. 


\section{$\S 4$. Symplectic structure on the space of representations}

On the space $\mathcal{R}$ of representations there is defined a natural (complex) symplectic structure that is induced by Poincaré duality (see Iwasaki [7]). This duality theorem in particular asserts that there is a nondegenerate pairing

$$
H^{1}\left(X_{0}, \mathcal{F}\left(\operatorname{Ad} E_{0}^{-}\right)\right) \otimes H_{c}^{1}\left(X_{0}, \mathcal{F}\left(\operatorname{Ad} E_{0}^{-}\right)\right) \longrightarrow \mathbb{C}
$$

which associates to cohomology classes

$$
[\Psi] \in H^{1}\left(X_{0}, \mathcal{F}\left(\operatorname{Ad} E_{0}^{-}\right)\right) \quad \text { and } \quad[\Theta] \in H_{c}^{1}\left(X_{0}, \mathcal{F}\left(\operatorname{Ad} E_{0}^{-}\right)\right)
$$

the complex constant

$$
\langle[\Psi],[\Theta]\rangle=\frac{1}{2 \pi \sqrt{-1}} \int_{X_{0}} \operatorname{tr}(\Psi \wedge \Theta),
$$

where $\Psi$ and $\Theta$ are representative closed forms under the isomorphisms (20).

With the same notation as in the previous section, let us consider the open subset $U=\bigcup_{k=0}^{2 m+1} U_{k}^{\prime}$ of $X_{0}$ with inclusion $j: U \rightarrow X_{0}$ and recall the diagram

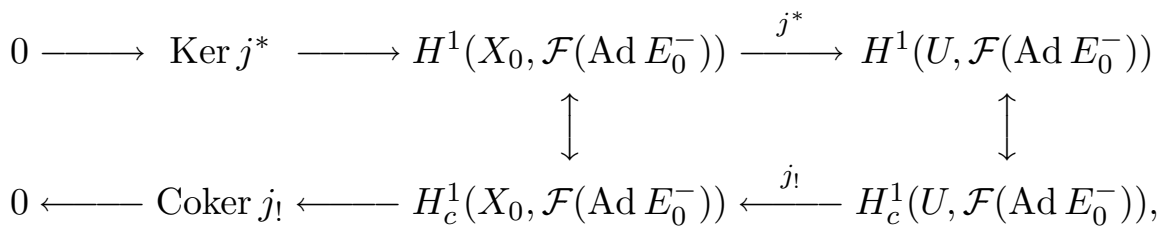

where the Poincaré-duality pairings are indicated by $\longleftrightarrow$. Since this diagram is commutative in the sense that

$$
\langle A, j ! B\rangle=\left\langle j^{*} A, B\right\rangle
$$

for all $A \in H^{1}\left(X_{0}, \mathcal{F}\left(\operatorname{Ad} E_{0}^{-}\right)\right)$and $B \in H_{c}^{1}\left(U, \mathcal{F}\left(\operatorname{Ad} E_{0}^{-}\right)\right.$) (recall the description of the mappings $j^{*}$ and $j$ ! in terms of differential forms), there arises, on the one hand, a canonical nondegenerate pairing

$$
\text { Ker } j^{*} \otimes \text { Coker } j ! \longrightarrow \mathbb{C}
$$

between the spaces Ker $j^{*}$ and Coker $j$. On the other hand, we have the following. 
LEMMA 2. The space Ker $j^{*}$ is canonically isomorphic to the space Coker $j$.

Proof. Let $A=[\Psi]$ be an element of Ker $j^{*}$ that is represented by a closed 1-form $\Psi \in \Gamma\left(X_{0}, \mathcal{E}^{1}\left(\operatorname{Ad} E_{0}^{-}\right)\right)$; thus the restriction $\Psi \mid U$ of $\Psi$ to $U$ has the form

$$
\Psi \mid U=\widehat{\nabla}_{0} T
$$

for some $T \in \Gamma\left(U, \mathcal{E}^{0}\left(\operatorname{Ad} E_{0}^{-}\right)\right)$. To construct the corresponding element of Coker $j$ ! under the desired isomorphism, we shall cut off the 1-form $\Psi$ in each neighborhood of the points $\left[a_{k}(0)\right](k=0, \ldots, 2 m+1)$. In each $U_{k} \subset M_{0}$, we take small open disks $U_{k}^{(1)} \subset U_{k}^{(0)} \subset U_{k}$ both centered at the point $\left[a_{k}(0)\right]$, and select a $C^{\infty}$ function $\sigma$ on $U=\bigcup_{k=0}^{2 m+1} U_{k}^{\prime}$ such that

$$
\begin{cases}\sigma=1 & \text { in } \frac{U_{k} \backslash U_{k}^{(0)} \quad(k=0, \ldots, 2 m+1),}{\sigma=0} \quad \text { in } U_{k}^{(1)} \backslash\left\{\left[a_{k}(0)\right]\right\} \quad(k=0, \ldots, 2 m+1) .\end{cases}
$$

Then, multiplying the cross-section $T$ in (29) by $\sigma$, we obtain another closed 1-form $\Psi^{-} \in \Gamma_{c}\left(X_{0}, \mathcal{E}^{1}\left(\operatorname{Ad} E_{0}^{-}\right)\right)$such that

$$
\begin{cases}\Psi^{-}=\Psi & \text { in } \frac{X_{0} \backslash \bigcup_{k=0}^{2 m+1} U_{k}^{(0)}}{\Psi^{(1)}} \backslash\left\{\left[a_{k}(0)\right]\right\} \quad(k=0, \ldots, 2 m+1) .\end{cases}
$$

To verify the well-definedness of this cutting-off procedure, suppose now that $\Psi^{--} \in \Gamma_{c}\left(X_{0}, \mathcal{E}^{1}\left(\operatorname{Ad} E_{0}^{-}\right)\right)$is another closed 1-form having the same property as $\Psi^{-}$. It then follows that the difference $\Psi^{-}-\Psi^{--}$has compact support in $U$, hence that the cohomology classes $\left[\Psi^{-}\right],\left[\Psi^{--}\right] \in H_{c}^{1}\left(X_{0}\right.$, $\left.\mathcal{F}\left(\operatorname{Ad} E_{0}^{-}\right)\right)$determine the same element of the quotient space

$$
\text { Coker } j_{!}=H_{c}^{1}\left(X_{0}, \mathcal{F}\left(\operatorname{Ad} E_{0}^{-}\right)\right) / j_{!} H_{c}^{1}\left(U, \mathcal{F}\left(\operatorname{Ad} E_{0}^{-}\right)\right) .
$$

Furthermore, a similar argument shows that this procedure is also independent of the choice of the representative 1-form $\Psi \in \Gamma\left(X_{0}, \mathcal{E}^{1}\left(\operatorname{Ad} E_{0}^{-}\right)\right)$; and consequently we have obtained a well-defined mapping

$$
\begin{gathered}
\operatorname{Ker} j^{*} \longrightarrow \text { Coker } j ! \\
{[\Psi] \longmapsto\left[\Psi^{-}\right]}
\end{gathered}
$$

It is a straightforward matter to verify that this mapping is indeed an isomorphism, and that completes the proof of the lemma. 
Combining the isomorphism $\operatorname{Ker} j^{*} \cong$ Coker $j$ ! with the pairing (28) now establishes a (skew-symmetric) nondegenerate bilinear form

$$
\operatorname{Ker} j^{*} \otimes \operatorname{Ker} j^{*} \longrightarrow \mathbb{C}
$$

on $\operatorname{Ker} j^{*}$; and via the natural identification

$$
T_{[\rho(0)]} \mathcal{R} \cong \operatorname{Ker} j^{*}: H^{1}\left(X_{0}, \mathcal{F}\left(\operatorname{Ad} E_{0}^{-}\right)\right) \longrightarrow H^{1}\left(U, \mathcal{F}\left(\operatorname{Ad} E_{0}^{-}\right)\right)
$$

(recall the discussion in Section 3.3), there then arises a nondegenerate 2 -form $\omega$ on the complex manifold $\mathcal{R}$. Since it can be shown that this 2 form is closed (see Goldman [2]), we obtain a natural (complex) symplectic structure on the space $\mathcal{R}$.

\section{$\S 5$. Evaluation of the symplectic form}

We have described the infinitesimal variation of the monodromy representations of the equations $(\tau(s), t(s), \lambda(s), \mu(s))$ at $s=0$ as a tangent vector to the manifold $\mathcal{R}$ and then recalled the description of the symplectic structure $\omega$ on $\mathcal{R}$. In this section we consider another family of equations passing through $(\tau(0), t(0), \lambda(0), \mu(0))$ and evaluate the symplectic form $\omega$ for the resulting two tangent vectors.

\subsection{Construction of diffeomorphisms}

Before turning to the evaluation, we construct a diffeomorphism $u_{s}$ : $\mathbb{C} \rightarrow \mathbb{C}$ satisfying (17). For this we choose a point $e \in \mathbb{C}$ such that $e \not \equiv a_{k}(0)$ $\bmod \Gamma_{0}$ for $k=0, \ldots, 2 m+1$ (see $(24)$ ), and construct a meromorphic function $f_{s}$ on $\mathbb{C}$ having poles only at points congruent to $e$ and satisfying

$$
\left\{\begin{array}{l}
f_{s}(z+1)=f_{s}(z)+1 \\
f_{s}(z+\tau(0))=f_{s}(z)+\tau(s)
\end{array}\right.
$$

for all $z \in \mathbb{C} \backslash\{$ poles\}. Now, differentiating the equalities in (32) with respect to $z$ implies that the function $f_{s}$ must be an indefinite integral of an elliptic function with fundamental periods $1, \tau(0)$; and we assume here that it has the form

$$
f_{s}(z)=\kappa(s) z+\epsilon(s) \zeta(z-e, \tau(0))
$$

for some holomorphic functions $\kappa(s), \epsilon(s)$ in $s$. Then, substituting this expression into (32) and using the Legendre relation $\eta_{2}(\tau(0))=\eta_{1}(\tau(0)) \tau(0)-$ 
$2 \pi \sqrt{-1}$ yields

$$
\left[\begin{array}{c}
\kappa(s) \\
\epsilon(s)
\end{array}\right]=-\frac{1}{2 \pi \sqrt{-1}}\left[\begin{array}{c}
\eta_{2}(\tau(0))-\eta_{1}(\tau(0)) \tau(s) \\
\tau(s)-\tau(0)
\end{array}\right]
$$

leading to the desired function $f_{s}$. (The term $\eta_{2}(\tau(0))$ is defined by $\zeta(z+$ $\tau(0), \tau(0))-\zeta(z, \tau(0))=\eta_{2}(\tau(0))$.) It should be observed here that

$$
\kappa(s) \rightarrow 1 \text { and } \quad \epsilon(s) \rightarrow 0 \quad \text { as } s \rightarrow 0
$$

To construct the desired diffeomorphism $u_{s}: \mathbb{C} \rightarrow \mathbb{C}$ satisfying (17), we use the cutting-off technique again for the function $f_{s}$ in a neighborhood of the point $e$ (recall the proof of Lemma 2). To this end we take sufficiently small open disks $V^{(1)} \subset V^{(0)} \subset V$ each centered at $e$, and select a $C^{\infty}$ function $\sigma$ on $\mathbb{C}$ such that

$$
\begin{cases}\sigma=1 & \text { in } \mathbb{C} \backslash\left(\Gamma_{0}\left(V^{(0)}\right)\right) \\ \sigma=0 & \text { in } \Gamma_{0}\left(\overline{V^{(1)}}\right), \\ \sigma(z+l+n \tau(0))=\sigma(z) & \text { for all } z \in \mathbb{C},(l, n) \in \mathbb{Z}^{2}\end{cases}
$$

Then, selecting another (uniquely determined) $C^{\infty}$ function $\chi_{s}$ on $\mathbb{C}$ (depending holomorphically on $s$ and) satisfying

$$
\begin{cases}\chi_{s}=1 & \text { in } \mathbb{C} \backslash\left(\Gamma_{0}\left(V^{(0)}\right)\right), \\ \chi_{s}=1 & \text { in } V\end{cases}
$$

we find that the function $u_{s}$ defined by

$$
u_{s}(z)=\chi_{s}(z) \kappa(s) z+\sigma(z) \epsilon(s) \zeta(z-e, \tau(0))
$$

satisfies (17), as desired. Note that $\chi_{s}$ has constantly value one in any fundamental parallelogram containing the open disk $V$, and in particular from (17), (33) that $\chi_{0}(z)=1$ for all $z \in \mathbb{C}$. Consequently, in view of (33), the function $u_{s}$ thus defined becomes indeed a diffeomorphism (for $s$ near 0 ), and moreover it is locally biholomorphic outside the disks $\Gamma_{0}\left(V^{(0)}\right)$. (In the argument below we shall consider $V$ also as an open subset of $X_{0}=M_{0} \backslash D_{0}$ that is disjoint from the punctured disks $U_{k}^{\prime}(k=0, \ldots, 2 m+1)$.) 


\subsection{Evaluation of the symplectic form}

Let us now consider another family $\left(\tau^{2}(s), t^{2}(s), \lambda^{2}(s), \mu^{2}(s)\right)$ of differential equations passing through the point $(\tau(0), t(0), \lambda(0), \mu(0))$ at $s=0$ and write the original family as $\left(\tau^{1}(s), t^{1}(s), \lambda^{1}(s), \mu^{1}(s)\right)$. Applying the geometric formulation also to the second family, we thus obtain the following objects $(n=1,2)$ :

$$
\begin{aligned}
& \Omega_{s}^{n}=\left(\begin{array}{cc}
0 & -1 \\
-q_{s}^{n}(z) & 0
\end{array}\right) d z, \\
& {\left[\begin{array}{c}
\kappa^{n}(s) \\
\epsilon^{n}(s)
\end{array}\right]=-\frac{1}{2 \pi \sqrt{-1}}\left[\begin{array}{c}
\eta_{2}(\tau(0))-\eta_{1}(\tau(0)) \tau^{n}(s) \\
\tau^{n}(s)-\tau(0)
\end{array}\right],} \\
& u_{s}^{n}(z)=\chi_{s}^{n}(z) \kappa^{n}(s) z+\sigma(z) \epsilon^{n}(s) \zeta(z-e, \tau(0)), \\
& \Omega_{s}^{n *}=\left(u_{s}^{n}\right)^{*} \Omega_{s}^{n},
\end{aligned}
$$

where $q_{s}^{n}(z)$ is given by substituting $(\tau, t, \lambda, \mu)=\left(\tau^{n}(s), t^{n}(s), \lambda^{n}(s), \mu^{n}(s)\right)$, $L=L^{n}(s)$ and $H_{i}=H_{i}^{n}(s)$ into $q(z)$ of $(2)$, and $\chi_{s}^{n}$ is the uniquely determined $C^{\infty}$ function on $\mathbb{C}$ that makes the $u_{s}^{n}$ as desired. The infinitesimal variation of the monodromy representations of the family $\left(\tau^{n}(s), t^{n}(s)\right.$, $\left.\lambda^{n}(s), \mu^{n}(s)\right)$ at $s=0$ is then described by the cohomology class $\left[\left(-\dot{\Omega}_{0}^{n *}\right)\right] \in$ $\operatorname{Ker} j^{*}$ (see (31)); and our goal in this section is to write out the complex number $\left\langle\left[\left(-\dot{\Omega}_{0}^{1 *}\right)\right],\left[\left(-\dot{\Omega}_{0}^{2 *}\right)\right]\right\rangle$ associated to these cohomology classes by the bilinear form (30).

For that purpose we first apply (19) to $\Omega_{s}^{n}=A_{s}^{n}(z) d z$ and $u_{s}^{n}$ above and obtain

$$
\dot{\Omega}_{0}^{n *}=\left[\begin{array}{cc}
0 & -d\left(\dot{u}_{0}^{n}(z)\right) \\
\left(-\dot{q}_{0}^{n}(z)-q_{0}^{n \prime}(z) \dot{u}_{0}^{n}(z)\right) d z-q_{0}^{n}(z) d\left(\dot{u}_{0}^{n}(z)\right) & 0
\end{array}\right] .
$$

Next, recalling that the bilinear form (30) was established by combining the isomorphism Ker $j^{*} \cong$ Coker $j$ ! of Lemma 2 with the pairing (28), we apply the cutting-off procedure of that lemma to the 1-form $\left(-\Omega_{0}^{2 *}\right) \in$ $\Gamma\left(X_{0}, \mathcal{E}^{1}\left(\operatorname{Ad} E_{0}^{-}\right)\right)$and obtain another closed 1-form $\left(-\Omega_{0}^{2 *-}\right) \in \Gamma_{c}\left(X_{0}\right.$, $\left.\mathcal{E}^{1}\left(\operatorname{Ad} E_{0}^{-}\right)\right)$satisfying

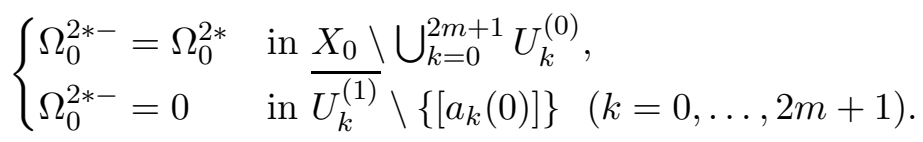

Then, by definition, we have

$$
\left\langle\left[\left(-\dot{\Omega}_{0}^{1 *}\right)\right],\left[\left(-\dot{\Omega}_{0}^{2 *}\right)\right]\right\rangle=\frac{1}{2 \pi \sqrt{-1}} \int_{X_{0}} \operatorname{tr}\left(\left(-\dot{\Omega}_{0}^{1 *}\right) \wedge\left(-\dot{\Omega}_{0}^{2 *-}\right)\right) .
$$


However, in view of (37), we find from (34)-(36) and (38) that the 1-forms $\left(-\dot{\Omega}_{0}^{1 *}\right)$ and $\left(-\dot{\Omega}_{0}^{2 *-}\right)$ are both of type $(1,0)$ outside the disk $V^{(0)}$ and the punctured disks $U_{k}^{(0)} \backslash\left\{\left[a_{k}(0)\right]\right\} \quad(k=0, \ldots, 2 m+1)$, hence that $\operatorname{tr}\left(\left(-\dot{\Omega}_{0}^{1 *}\right) \wedge\left(-\dot{\Omega}_{0}^{2 *-}\right)\right)=0$ there. (These disks are now viewed as open subsets of $X_{0}$.) Moreover, it follows from the discussion in Section 3.3 that there exist $C^{\infty}$ cross-sections $T \in \Gamma\left(V, \mathcal{E}^{0}\left(\operatorname{Ad} E_{0}^{-}\right)\right)$and $T_{k} \in \Gamma\left(U_{k}^{\prime}, \mathcal{E}^{0}\left(\operatorname{Ad} E_{0}^{-}\right)\right)$ $(k=0, \ldots, 2 m+1)$ satisfying

$$
\begin{cases}\widehat{\nabla}_{0} T=-\dot{\Omega}_{0}^{1 *} & \text { in } V \\ \widehat{\nabla}_{0} T_{k}=-\dot{\Omega}_{0}^{1 *} & \text { in } U_{k}^{\prime} \quad(k=0, \ldots, 2 m+1) ;\end{cases}
$$

indeed, in view of (27) and (36), it suffices to set

$$
\left\{\begin{array}{l}
T=\dot{Y}(z, 0) Y(z, 0)^{-1}+Y^{\prime}(z, 0) Y(z, 0)^{-1} \dot{u}_{0}^{1}(z), \\
T_{k}=\dot{Y}_{k}(z, 0) Y_{k}(z, 0)^{-1}+Y_{k}^{\prime}(z, 0) Y_{k}(z, 0)^{-1} \dot{u}_{0}^{1}(z),
\end{array}\right.
$$

where the $Y(z, s)$ and $Y_{k}(z, s)$ are fundamental solutions of the system $d y+\Omega_{s}^{1} y=0$ around $z=e$ and $z=a_{k}^{1}(s)$ respectively that are chosen to have constant Wronskians with respect to both $z$ and $s$, and also to have the form (25) for $k=0, \ldots, 2 m+1$ (see the proof of Lemma 1). (We use the notation $a_{k}^{1}(s)$ in an obvious manner.) Consequently, in view of (38) again, we have

$$
\begin{aligned}
& \left\langle\left[\left(-\dot{\Omega}_{0}^{1 *}\right)\right],\left[\left(-\dot{\Omega}_{0}^{2 *}\right)\right]\right\rangle \\
& =\frac{1}{2 \pi \sqrt{-1}}\left[\int_{V^{(0)}} \operatorname{tr}\left(\left(\widehat{\nabla}_{0} T\right) \wedge\left(-\dot{\Omega}_{0}^{2 *-}\right)\right)\right. \\
& \left.\quad+\sum_{k=0}^{2 m+1} \int_{U_{k}^{(0)} \backslash U_{k}^{(1)}} \operatorname{tr}\left(\left(\widehat{\nabla}_{0} T_{k}\right) \wedge\left(-\dot{\Omega}_{0}^{2 *-}\right)\right)\right] \\
& =\frac{1}{2 \pi \sqrt{-1}}\left[\int_{V^{(0)}} d \operatorname{tr}\left(T\left(-\dot{\Omega}_{0}^{2 *-}\right)\right)+\sum_{k=0}^{2 m+1} \int_{\overline{U_{k}^{(0)}} \backslash U_{k}^{(1)}} d \operatorname{tr}\left(T_{k}\left(-\dot{\Omega}_{0}^{2 *-}\right)\right)\right] \\
& =\frac{1}{2 \pi \sqrt{-1}}\left[\int_{\partial \overline{V^{(0)}}} \operatorname{tr}\left(T\left(-\dot{\Omega}_{0}^{2 *}\right)\right)+\sum_{k=0}^{2 m+1} \int_{\frac{\partial U_{k}^{(0)}}{}} \operatorname{tr}\left(T_{k}\left(-\dot{\Omega}_{0}^{2 *}\right)\right)\right],
\end{aligned}
$$

where we have used the closedness of the 1 -form $-\dot{\Omega}_{0}^{2 *-}$ at the second equality, and formula (38) and Stokes' theorem at the final equality.

Now, looking at the final line of this last formula, we note from (39), (37) and (36) that the $T_{k}$ and $-\dot{\Omega}_{0}^{2 *}$ are both meromorphic in $U_{k}$ with at 
most a pole at $z=a_{k}(0)$ whereas the $T$ and $-\dot{\Omega}_{0}^{2 *}$ cannot be holomorphic (nor meromorphic) in $V$ because of the appearance of the terms $\dot{u}_{0}^{n}(z)(n=$ $1,2)$ respectively. However, if we set

$$
\begin{aligned}
& f_{s}^{n}(z)=\kappa^{n}(s) z+\epsilon^{n}(s) \zeta(z-e, \tau(0)), \\
& T^{+}=\dot{Y}(z, 0) Y(z, 0)^{-1}+Y^{\prime}(z, 0) Y(z, 0)^{-1} \dot{f}_{0}^{1}(z), \\
& \dot{\Omega}_{0}^{n *+}=\left[\begin{array}{cc}
0 & -d\left(\dot{f}_{0}^{n}(z)\right) \\
\left(-\dot{q}_{0}^{n}(z)-q_{0}^{n \prime}(z) \dot{f}_{0}^{n}(z)\right) d z-q_{0}^{n}(z) d\left(\dot{f}_{0}^{n}(z)\right) & 0
\end{array}\right],
\end{aligned}
$$

then $T^{+}$and $-\dot{\Omega}_{0}^{n *+}$ become both meromorphic in $V$ with at most a pole at $z=e$, and they satisfy $T^{+}=T$ and $-\dot{\Omega}_{0}^{n *+}=-\dot{\Omega}_{0}^{n *}$ respectively in $V \backslash V^{(0)}$ (see (34)-(36)). Therefore, putting this all together, we obtain from the residue theorem

$$
\left\langle\left[\left(-\dot{\Omega}_{0}^{1 *}\right)\right],\left[\left(-\dot{\Omega}_{0}^{2 *}\right)\right]\right\rangle=\operatorname{Res}_{z=e} \operatorname{tr}\left(T^{+}\left(-\dot{\Omega}_{0}^{2 *+}\right)\right)+\sum_{k=0}^{2 m+1} \underset{z=a_{k}(0)}{\operatorname{Res}} \operatorname{tr}\left(T_{k}\left(-\dot{\Omega}_{0}^{2 *}\right)\right) .
$$

Remark. Formula (41) is a generalization of Iwasaki's result [7, Lemma 6]. He obtained his main result by carrying out residue calculus explicitly in terms of the parameters of the linear equations in consideration. Our goal will be to apply this method to our case of varying elliptic curves and vector bundles.

\subsection{Third-order equation}

Finally, for use in the next section, we write out the equations

$$
\begin{cases}\widehat{\nabla}_{0} T^{+}=-\dot{\Omega}_{0}^{1 *+} & \text { in } V \\ \widehat{\nabla}_{0} T_{k}=-\dot{\Omega}_{0}^{1 *} & \text { in } U_{k}(k=0, \ldots, 2 m+1)\end{cases}
$$

for the cross-sections $T^{+} \in \Gamma\left(V, \mathcal{M}\left(\operatorname{Ad} E_{0}\right)\right)$ and $T_{k} \in \Gamma\left(U_{k}, \mathcal{M}\left(\operatorname{Ad} E_{0}\right)\right)$ in detail, where $\mathcal{M}\left(\mathrm{Ad} E_{0}\right)$ denotes the sheaf of germs of meromorphic crosssections of the bundle Ad $E_{0}$. (The computation presented below is due to M. Furuta.) Setting

$$
T^{+}=\left[\begin{array}{cc}
g(z) & h(z) \\
l(z) & -g(z)
\end{array}\right]
$$

and recalling that the connection $\widehat{\nabla}_{0}$ takes the form $\widehat{\nabla}_{0}=d+\left[\Omega_{0}^{1}, \cdot\right]$ (note that $\Omega_{0}^{1}=\Omega_{0}^{2}$, we find that the first equation in (42) reduces to the following 
formulas:

$$
\left\{\begin{array}{l}
g(z)=\frac{1}{2}\left[\dot{f}_{0}^{1}(z)-h(z)\right]^{\prime} \\
l(z)=\frac{1}{2}\left[\dot{f}_{0}^{1}(z)-h(z)\right]^{\prime \prime}+q_{0}^{1}(z) h(z) \\
h(z)=v(z)+\dot{f}_{0}^{1}(z)
\end{array}\right.
$$

where the function $v(z)$ is a solution of the third-order equation

$$
-\frac{1}{2} v^{\prime \prime \prime}(z)+2 q_{0}^{1}(z) v^{\prime}(z)+q_{0}^{1 \prime}(z) v(z)=\dot{q}_{0}^{1}(z, 0) .
$$

Similarly, by setting

$$
T_{k}=\left[\begin{array}{cc}
g_{k}(z) & h_{k}(z) \\
l_{k}(z) & -g_{k}(z)
\end{array}\right],
$$

the second equation in (42) is written out explicitly as

$$
\left\{\begin{array}{l}
g_{k}(z)=\frac{1}{2}\left[\dot{f}_{0}^{1}(z)-h_{k}(z)\right]^{\prime} \\
l_{k}(z)=\frac{1}{2}\left[\dot{f}_{0}^{1}(z)-h_{k}(z)\right]^{\prime \prime}+q_{0}^{1}(z) h_{k}(z) \\
h_{k}(z)=v_{k}(z)+\dot{f}_{0}^{1}(z)
\end{array}\right.
$$

where the function $v_{k}(z)$ is a solution of the equation

$$
-\frac{1}{2} v_{k}^{\prime \prime \prime}(z)+2 q_{0}^{1}(z) v_{k}^{\prime}(z)+q_{0}^{1 \prime}(z) v_{k}(z)=\dot{q}_{0}^{1}(z, 0),
$$

which has its (regular) singularity at $z=a_{k}(0)$. (Note that $\dot{u}_{0}^{1}(z)=\dot{f}_{0}^{1}(z)$ in $U_{k}$.)

Now we conclude this section with the following technical lemma.

LEMma 3. For any fundamental solution $Y(z, s)$ of the system $d y+$ $\Omega_{s}^{1} y=0$ at $z=e$ that is chosen to have constant Wronskian with respect to both $z$ and $s$, the function $v(z)$ defined by (40), (43) and (44) is holomorphic in the disk $V$. Similarly, for any fundamental solution $Y_{k}(z, s)$ of that system at $z=a_{k}^{1}(s)$ that is chosen to have constant Wronskian with respect to both $z$ and $s$ and also to have the form (25), the function $v_{k}(z)$ defined by (39), (46) and (47) is holomorphic in the disk $U_{k}(k=0, \ldots, 2 m+1)$. 
Proof. To verify the second half of the lemma first, let us take a fundamental system $\left(w^{1}(z, s), w^{2}(z, s)\right)$ of solutions of the equation

$$
\frac{d^{2} w}{d z^{2}}=q_{s}^{1}(z) w
$$

at $z=a_{k}^{1}(s)$ that has the explicit form

$$
\left\{\begin{array}{l}
w^{1}(z, s)=\left(z-a_{k}^{1}(s)\right)^{\left(1+\theta_{k}\right) / 2} \varphi(z, s), \\
w^{2}(z, s)=\left(z-a_{k}^{1}(s)\right)^{\left(1-\theta_{k}\right) / 2} \psi(z, s),
\end{array}\right.
$$

where the $\varphi(z, s)$ and $\psi(z, s)$ are holomorphic in both $z$ and $s$, and $\varphi\left(a_{k}^{1}(s), s\right) \neq 0, \psi\left(a_{k}^{1}(s), s\right) \neq 0$; furthermore we have set $\theta_{k}=\theta_{i}$ for $k=i=0, \ldots, m$ and $\theta_{k}=2$ for $k=m+1, \ldots, 2 m+1$. (Recall the assumptions on the singular points of our equations (see Section 1.1 and Iwasaki et al. [8, pp. 163, 183-184]).) The matrix

$$
Y_{k}(z, s)=\left[\begin{array}{cc}
w^{1}(z, s) & w^{2}(z, s) \\
w^{1 \prime}(z, s) & w^{2 \prime}(z, s)
\end{array}\right]
$$

then serves as a fundamental solution of the system $d y+\Omega_{s}^{1} y=0$ having the form (25); and conversely any such fundamental solution of that system gives in an obvious manner a fundamental system of (49) having the form (50). Now, by multiplying the $w^{1}$ and $w^{2}$ by nowhere-vanishing holomorphic functions in $s$ if necessary, we assume here that the Wronskian of $Y_{k}(z, s)$ is constant with respect to $z$ and $s$. By an easy calculation we then find that the $(1,2)$-component $h_{k}(z)$ of the matrix $T_{k}$ defined by (39) equals

$$
c^{-1}\left[-\dot{w}^{1}(z, 0) w^{2}(z, 0)+\dot{w}^{2}(z, 0) w^{1}(z, 0)\right]+\dot{f}_{0}^{1}(z),
$$

where $c$ denotes the constant Wronskian of $Y_{k}(z, s)$, so that

$$
v_{k}(z)=c^{-1}\left[-\dot{w}^{1}(z, 0) w^{2}(z, 0)+\dot{w}^{2}(z, 0) w^{1}(z, 0)\right]
$$

by (47). However, substituting the explicit form (50) into the right-hand side of this formula now yields

$$
\begin{aligned}
v_{k}(z)=c^{-1}\left(z-a_{k}^{1}(0)\right)[-\dot{\varphi}(z, 0) \psi(z, 0)+\dot{\psi} & (z, 0) \varphi(z, 0)] \\
& +c^{-1} \theta_{k} \dot{a}_{k}^{1}(0) \varphi(z, 0) \psi(z, 0)
\end{aligned}
$$

and therefore the function $v_{k}(z)$ is indeed holomorphic in the sufficiently small disk $U_{k}$, as desired. Arguing the same way also establishes the result for $v(z)$, and the proof is thereby concluded. 
Remarks. This result should be compared to Iwasaki [7, p. 339], where he examines the order of an arbitrary meromorphic function satisfying the third-order equation (48) and concludes that it is either 0 or -1 . We also note that the requirement that the matrix $Y_{k}(z, s)$ have constant Wronskian independent of $z$ and $s$ is crucial for the holomorphicity of the function $v_{k}(z)$. See Iwasaki et al. [8, Lemma 4.4.2].

\section{§6. Residue calculus}

In this section we carry out residue calculus for formula (41) and obtain an expression in terms of the local coordinates $(\tau, t, \lambda, \mu)$ of $\mathcal{E}$, thus completing the proof of Theorem 1. Our method is basically the same as that of Iwasaki [7].

\subsection{First term}

To compute $\operatorname{Res}_{z=e} \operatorname{tr}\left(T^{+}\left(-\dot{\Omega}_{0}^{2 *+}\right)\right)$, write the terms $T^{+}$and $-\dot{\Omega}_{0}^{2 *+}$ as in (43) and (40). We then find from (44) and the equality $q_{0}^{1}(z)=q_{0}^{2}(z)$ that

$$
\begin{aligned}
\operatorname{tr}\left(T^{+}\left(-\dot{\Omega}_{0}^{2 *+}\right)\right)=\left([ v ( z ) + \dot { f } _ { 0 } ^ { 1 } ( z ) ] \left[\dot{q}_{0}^{2}(z)+q_{0}^{2 \prime}(z) \dot{f}_{0}^{2}(z)\right.\right. & \\
& \left.\left.+2 q_{0}^{2}(z) \dot{f}_{0}^{2 \prime}(z)\right]-\frac{1}{2} v^{\prime \prime}(z) \dot{f}_{0}^{2 \prime}(z)\right) d z
\end{aligned}
$$

To find the desired residue, we expand out each term of this expression at $z=e$. First, since the functions $q_{s}^{n}(z)(n=1,2)$ are holomorphic in the disk $V$ (for $s$ near 0 ), their Taylor expansions at $z=e$ are of the form

$$
q_{s}^{n}(z)=c_{0}^{n}(s)+c_{1}^{n}(s)(z-e)+c_{2}^{n}(s)(z-e)^{2}+\text { higher terms }
$$

where the coefficients $c_{j}^{n}(s)$ depend holomorphically on $s$. (Note that $q_{0}^{1}(z)=q_{0}^{2}(z)$ implies $c_{j}^{1}(0)=c_{j}^{2}(0)$ for all $j$.) Second, we find from $(40)$ and (36) that the function $\dot{f}_{0}^{n}(z)$ is expanded out as

$$
\dot{f}_{0}^{n}(z)=-\frac{\dot{\tau}^{n}(0)}{2 \pi \sqrt{-1}}\left[\frac{1}{z-e}-\eta_{1}(\tau(0)) e-\eta_{1}(\tau(0))(z-e)+\text { higher terms }\right] .
$$

Third, recalling from Lemma 3 that the function $v(z)$ can be chosen to be holomorphic in $V$, let us expand it out as

$$
v(z)=\alpha+\beta(z-e)+\gamma(z-e)^{2}+\delta(z-e)^{3}+\text { higher terms. }
$$


Now, substituting (54) and (52) into (45) and comparing the constant terms of both sides, we obtain

$$
-3 \delta+2 c_{0}^{1}(0) \beta+c_{1}^{1}(0) \alpha=-3 \delta+2 c_{0}^{2}(0) \beta+c_{1}^{2}(0) \alpha=\dot{c}_{0}^{1}(0) .
$$

On the other hand, substituting (52)-(54) into the right-hand side of (51), we find

$\operatorname{Res}_{z=e} \operatorname{tr}\left(T^{+}\left(-\dot{\Omega}_{0}^{2 *+}\right)\right)=\frac{-1}{2 \pi \sqrt{-1}}\left[\left(-\alpha c_{1}^{2}(0)-2 \beta c_{0}^{2}(0)+3 \delta\right) \dot{\tau}^{2}(0)+\dot{\tau}^{1}(0) \dot{c}_{0}^{2}(0)\right]$

and consequently putting these last two formulas together yields

$$
\operatorname{Res}_{z=e} \operatorname{tr}\left(T^{+}\left(-\dot{\Omega}_{0}^{2 *+}\right)\right)=\frac{1}{2 \pi \sqrt{-1}}\left(\dot{c}_{0}^{1}(0) \dot{\tau}^{2}(0)-\dot{\tau}^{1}(0) \dot{c}_{0}^{2}(0)\right) .
$$

\subsection{Second term}

We compute $\operatorname{Res}_{z=a_{k}(0)} \operatorname{tr}\left(T_{k}\left(-\dot{\Omega}_{0}^{2 *}\right)\right)$ similarly. As before, we start with the formula (see (46), (37) and (47))

$$
\begin{aligned}
\operatorname{tr}\left(T_{k}\left(-\dot{\Omega}_{0}^{2 *}\right)\right)=\left([ v _ { k } ( z ) + \dot { f } _ { 0 } ^ { 1 } ( z ) ] \left[\dot{q}_{0}^{2}(z)+q_{0}^{2 \prime}(z) \dot{f}_{0}^{2}(z)\right.\right. & \\
+ & \left.\left.2 q_{0}^{2}(z) \dot{f}_{0}^{2 \prime}(z)\right]-\frac{1}{2} v_{k}^{\prime \prime}(z) \dot{f}_{0}^{2 \prime}(z)\right) d z
\end{aligned}
$$

and expand out each term of this expression at $z=a_{k}(0)$. First, in view of (2), the Laurent expansions of the $q_{s}^{n}(z)$ at $z=a_{k}^{n}(s)$ take the form

$$
\begin{aligned}
& q_{s}^{n}(z)=\frac{b_{k,-2}^{n}}{\left(z-a_{k}^{n}(s)\right)^{2}}+\frac{b_{k,-1}^{n}(s)}{z-a_{k}^{n}(s)}+b_{k, 0}^{n}(s)+b_{k, 1}^{n}(s)\left(z-a_{k}^{n}(s)\right) \\
& + \text { higher terms, } \\
& \left\{\begin{array}{r}
b_{k,-2}^{n}=\frac{1}{4}\left(\theta_{i}^{2}-1\right), b_{k,-1}^{n}(s)=H_{i}^{n}(s) \quad \text { for } k=i=0, \ldots, m, \\
b_{k,-2}^{n}=\frac{3}{4}, b_{k,-1}^{n}(s)=-\mu_{\alpha}^{n}(s) \\
\quad \text { for } k=\alpha+(m+1)=m+1, \ldots, 2 m+1 .
\end{array}\right.
\end{aligned}
$$

(Again we have $b_{k, j}^{1}(0)=b_{k, j}^{2}(0)$ for all $j$ because $q_{0}^{1}(z)=q_{0}^{2}(z)$.) Second, since the point $e$ is away from the disk $U_{k}$, we can expand out the function 
$\dot{f}_{0}^{n}(z)$ as

(58)

$$
\begin{aligned}
& \dot{f}_{0}^{n}(z)=-\frac{\dot{\tau}^{n}(0)}{2 \pi \sqrt{-1}}\left[r_{0}+r_{1}\left(z-a_{k}(0)\right)+r_{2}\left(z-a_{k}(0)\right)^{2}+\text { higher terms }\right] \\
& \left\{\begin{array}{l}
r_{0}=-\eta_{1}(\tau(0)) a_{k}(0)+\zeta\left(a_{k}(0)-e, \tau(0)\right), \\
r_{1}=-\eta_{1}(\tau(0))+\zeta^{\prime}\left(a_{k}(0)-e, \tau(0)\right), \\
r_{2}=\frac{1}{2} \zeta^{\prime \prime}\left(a_{k}(0)-e, \tau(0)\right)
\end{array}\right.
\end{aligned}
$$

Third, it follows from Lemma 3 that the function $v_{k}(z)$ is expanded out as

$$
v_{k}(z)=\alpha+\beta\left(z-a_{k}(0)\right)+\gamma\left(z-a_{k}(0)\right)^{2}+\text { higher terms. }
$$

Then, substituting (59) and (57) into (48) and comparing the principal parts yields

$$
\alpha=-\dot{a}_{k}^{1}(0), \quad 2 b_{k,-2}^{2} \gamma+b_{k,-1}^{2}(0) \beta=\dot{b}_{k,-1}^{1}(0)
$$

where we have used $b_{k,-2}^{n} \neq 0$ and $b_{k, j}^{1}(0)=b_{k, j}^{2}(0)$. On the other hand, we substitute (57)-(59) into the right-hand side of (56) and then find that

$$
\begin{aligned}
& \underset{z=a_{k}(0)}{\operatorname{Res}} \operatorname{tr}\left(T_{k}\left(-\dot{\Omega}_{0}^{2 *}\right)\right) \\
& =\frac{-1}{2 \pi \sqrt{-1}}\left\{\left[\alpha\left(2 b_{k,-2}^{2} r_{2}+b_{k,-1}^{2}(0) r_{1}\right)-\left(\beta b_{k,-1}^{2}(0)+2 \gamma b_{k,-2}^{2}\right) r_{0}\right] \dot{\tau}^{2}(0)\right. \\
& \left.\quad+\dot{\tau}^{1}(0)\left[r_{0} \dot{b}_{k,-1}^{2}(0)+r_{1} b_{k,-1}^{2}(0) \dot{a}_{k}^{2}(0)+2 r_{2} b_{k,-2}^{2} \dot{a}_{k}^{2}(0)\right]\right\} \\
& \quad+\alpha \dot{b}_{k,-1}^{2}(0)+\left(\beta b_{k,-1}^{2}(0)+2 \gamma b_{k,-2}^{2}\right) \dot{a}_{k}^{2}(0) .
\end{aligned}
$$

\subsection{Proof of Theorem 1}

At this point, to write out the term $\dot{c}_{0}^{n}(0)$ in (55) in detail, we note that in terms of the notation in (57), the function $q_{s}^{n}(z)$ is written explicitly as $q_{s}^{n}(z)=L^{n}(s)+\sum_{k=0}^{2 m+1}\left[b_{k,-1}^{n}(s) \zeta\left(z-a_{k}^{n}(s), \tau^{n}(s)\right)+b_{k,-2}^{n} \wp\left(z-a_{k}^{n}(s), \tau^{n}(s)\right)\right]$. 
Since the $c_{0}^{n}(s)$ are the constant terms of the Taylor expansions (52), we then have

$$
\begin{aligned}
\dot{c}_{0}^{n}(0)= & \dot{L}^{n}(0)+\sum_{k=0}^{2 m+1}\left\{\dot{b}_{k,-1}^{n}(0) \zeta\left(e-a_{k}(0), \tau(0)\right)\right. \\
& +b_{k,-1}^{n}(0)\left[\zeta^{\prime}\left(e-a_{k}(0), \tau(0)\right)\left(-\dot{a}_{k}^{n}(0)\right)+\frac{\partial \zeta}{\partial \tau}\left(e-a_{k}(0), \tau(0)\right) \dot{\tau}^{n}(0)\right] \\
& \left.+b_{k,-2}^{n}\left[\wp^{\prime}\left(e-a_{k}(0), \tau(0)\right)\left(-\dot{a}_{k}^{n}(0)\right)+\frac{\partial \wp}{\partial \tau}\left(e-a_{k}(0), \tau(0)\right) \dot{\tau}^{n}(0)\right]\right\} .
\end{aligned}
$$

In summary of all this, substituting (62) into (55), and (60), (58) into (61) respectively, we finally conclude from (41) that

$$
\begin{aligned}
& \left\langle\left[\left(-\dot{\Omega}_{0}^{1 *}\right)\right],\left[\left(-\dot{\Omega}_{0}^{2 *}\right)\right]\right\rangle \\
& =\sum_{k=0}^{2 m+1}\left[\dot{b}_{k,-1}^{1}(0) \dot{a}_{k}^{2}(0)-\dot{b}_{k,-1}^{2}(0) \dot{a}_{k}^{1}(0)\right] \\
& \quad+\frac{1}{2 \pi \sqrt{-1}}\left[\dot{L}^{1}(0) \dot{\tau}^{2}(0)-\dot{L}^{2}(0) \dot{\tau}^{1}(0)\right] \\
& \quad-\frac{\eta_{1}(\tau(0))}{2 \pi \sqrt{-1}} \sum_{k=0}^{2 m+1}\left\{\left[a_{k}(0) \dot{b}_{k,-1}^{1}(0)+b_{k,-1}^{1}(0) \dot{a}_{k}^{1}(0)\right] \dot{\tau}^{2}(0)\right. \\
& \left.\quad-\left[a_{k}(0) \dot{b}_{k,-1}^{2}(0)+b_{k,-1}^{2}(0) \dot{a}_{k}^{2}(0)\right] \dot{\tau}^{1}(0)\right\},
\end{aligned}
$$

where we have used the equality $b_{k, j}^{1}(0)=b_{k, j}^{2}(0)$ again; and consequently, recalling the original notation for $a_{k}^{n}(s)$ and $b_{k,-1}^{n}(s)$, and in particular keeping in mind that we have always $t_{0}=d t_{0}=0$, we complete the proof of Theorem 1. (Note that (i) the $\zeta$-function $\zeta(z, \tau)$ is odd (with respect to $z$ ) whereas the $\wp$-function $\wp(z, \tau)$ is even, and that (ii) $d \eta_{1}(\tau) \wedge d \tau=0$.)

\section{$\S 7$. The null-distribution}

Taking a closer look at the explicit form (9) of the pulled-back 2-form $\mathcal{F}^{*} \omega$, let us move on to the description of the infinitesimal directions that make $\mathcal{F}^{*} \omega$ degenerate.

We begin by showing that the differential $d_{r} \mathcal{F}$ of $\mathcal{F}$ at an arbitrary point $r \in \mathcal{W}$ has maximal rank $2(m+1)$. To see this we first note the inequality $\operatorname{rank}\left(\mathcal{F}^{*} \omega\right)_{r} \leqq \operatorname{rank} d_{r} \mathcal{F}$. (This is an immediate consequence of the 
definition of the pulled-back form $\mathcal{F}^{*} \omega$. Recall that the rank of the 2-form $\mathcal{F}^{*} \omega$ at the point $r$ is precisely the rank of the linear mapping from $T_{r} \mathcal{W}$ to $T_{r}^{*} \mathcal{W}$ which is defined by $\xi \mapsto\left(\mathcal{F}^{*} \omega\right)_{r}(\cdot, \xi)$.) However, since the first term $-2\left(\sum_{\alpha=0}^{m} d \mu_{\alpha} \wedge d \lambda_{\alpha}\right)$ of $\mathcal{F}^{*} \omega$ is clearly nondegenerate on the (locally defined) $(\lambda, \mu)$-space, it follows that $2(m+1) \leqq \operatorname{rank}\left(\mathcal{F}^{*} \omega\right)_{r}$, which suffices to conclude the proof of the result. From this we then find that

(i) the fibers $\mathcal{F}^{-1}(\rho)$ of $\mathcal{F}$ are all smooth,

(ii) their connected components precisely serve as the maximal connected integral manifolds of the distribution $\mathcal{D}=\{\xi \in T \mathcal{W} ; d \mathcal{F}(\xi)=0\}$ of rank $m+1$,

(iii) the distribution $\mathcal{D}$ is also described as $\mathcal{D}=\left\{\xi \in T \mathcal{W} ; \mathcal{F}^{*} \omega(\cdot, \xi) \equiv 0\right\}$, namely the null-distribution of $\mathcal{F}^{*} \omega$ (see Section 1).

With these observations made, it is now quite straightforward to write out the null-distribution of $\mathcal{F}^{*} \omega$ explicitly. Indeed, from the nondegeneracy of the first term of $\mathcal{F}^{*} \omega$ again, it follows that any nonzero tangent vector $\xi$ satisfying $\mathcal{F}^{*} \omega(\cdot, \xi) \equiv 0$ must have a nonzero component in the $(\tau, t)$ directions. However, since we already know that the null-distribution $\mathcal{D}$ has rank $m+1$, which is exactly the dimension of the $(\tau, t)$-space, we then find that $\mathcal{D}$ must be spanned by the $m+1$ vector fields of the form

$$
\left\{\begin{array}{l}
\mathcal{H}_{i}=\frac{\partial}{\partial t_{i}}+\sum_{\alpha=0}^{m}\left(A_{\alpha}^{i} \frac{\partial}{\partial \lambda_{\alpha}}+B_{\alpha}^{i} \frac{\partial}{\partial \mu_{\alpha}}\right) \quad(i=1, \ldots, m) \\
\mathcal{H}_{\tau}=\frac{\partial}{\partial \tau}+\sum_{\alpha=0}^{m}\left(C_{\alpha} \frac{\partial}{\partial \lambda_{\alpha}}+D_{\alpha} \frac{\partial}{\partial \mu_{\alpha}}\right)
\end{array}\right.
$$

for some holomorphic functions $A_{\alpha}^{i}, B_{\alpha}^{i}, C_{\alpha}, D_{\alpha}$ in $(\tau, t, \lambda, \mu)$. Furthermore a simple calculation shows that the vectors $\mathcal{H}_{i}$ and $\mathcal{H}_{\tau}$ above make the 2 -form $\mathcal{F}^{*} \omega$ degenerate precisely when

$$
\left\{\begin{array}{l}
A_{\alpha}^{i}=\frac{\partial H_{i}}{\partial \mu_{\alpha}}, \quad B_{\alpha}^{i}=-\frac{\partial H_{i}}{\partial \lambda_{\alpha}} \\
\sum_{\alpha=0}^{m}\left(\frac{\partial H_{j}}{\partial \mu_{\alpha}} \frac{\partial H_{i}}{\partial \lambda_{\alpha}}-\frac{\partial H_{j}}{\partial \lambda_{\alpha}} \frac{\partial H_{i}}{\partial \mu_{\alpha}}\right)=\frac{\partial H_{j}}{\partial t_{i}}-\frac{\partial H_{i}}{\partial t_{j}} \quad(j=1, \ldots, m)
\end{array}\right.
$$


and

$$
\left\{\begin{array}{l}
C_{\alpha}=\frac{\partial K}{\partial \mu_{\alpha}}, \quad D_{\alpha}=-\frac{\partial K}{\partial \lambda_{\alpha}} \\
\sum_{\alpha=0}^{m}\left(\frac{\partial H_{j}}{\partial \mu_{\alpha}} \frac{\partial K}{\partial \lambda_{\alpha}}-\frac{\partial H_{j}}{\partial \lambda_{\alpha}} \frac{\partial K}{\partial \mu_{\alpha}}\right)=\frac{\partial H_{j}}{\partial \tau}-\frac{\partial K}{\partial t_{j}} \quad(j=1, \ldots, m)
\end{array}\right.
$$

respectively (see Manin [15, pp. 140-141]); and consequently we conclude that the null-distribution $\mathcal{D}$ has as a local basis the vector fields

$$
\left\{\begin{array}{l}
\mathcal{H}_{i}=\frac{\partial}{\partial t_{i}}+\sum_{\alpha=0}^{m}\left(\frac{\partial H_{i}}{\partial \mu_{\alpha}} \frac{\partial}{\partial \lambda_{\alpha}}-\frac{\partial H_{i}}{\partial \lambda_{\alpha}} \frac{\partial}{\partial \mu_{\alpha}}\right) \quad(i=1, \ldots, m), \\
\mathcal{H}_{\tau}=\frac{\partial}{\partial \tau}+\sum_{\alpha=0}^{m}\left(\frac{\partial K}{\partial \mu_{\alpha}} \frac{\partial}{\partial \lambda_{\alpha}}-\frac{\partial K}{\partial \lambda_{\alpha}} \frac{\partial}{\partial \mu_{\alpha}}\right) .
\end{array}\right.
$$

Now, since these vector fields just describe the time-evolution directions of the $\lambda_{\alpha}$ and $\mu_{\alpha}$ with respect to the time-parameters $t_{i}$ and $\tau$ with Hamiltonians $H_{i}$ and $K$ (see Iwasaki [6, p. 522] and [15, pp. 140-141]), we complete the proof of Theorem 2 .

Remark. In the discussion above we did not need to verify the validity of the third formulas of (63) and (64) nor even to examine the complex analytic behavior of the partial derivatives of the Hamiltonian functions $H_{i}$ and $K$ (compare with Okamoto [18, pp. 303-307] and [6, pp. 508-521]). This is an advantage of our geometric approach, which ensures the existence of integral manifolds of a specific dimension for the null-distribution $\mathcal{D}$.

\section{§8. The two-point case}

In this section we examine the simplest case $m=0$ of equation (1)(3) closely, and show that the resulting Hamiltonian system describing the isomonodromic deformations reduces to a special instance of the expression for the sixth Painlevé equation due to Manin [15].

When $m=0$, the coefficient $q(z)$ of equation (1)-(3) takes the simple form

$$
q(z)=L+H \zeta(z, \tau)+\frac{1}{4}\left(\theta^{2}-1\right) \wp(z, \tau)-\mu \zeta(z-\lambda, \tau)+\frac{3}{4} \wp(z-\lambda, \tau),
$$

where we dropped the suffix 0 for simplicity (recall the normalization $t_{0}=$ 0 ); and equation (6) (corresponding to assumption (A2) and equality (3)) 
reduces to

$$
\left\{\begin{array}{l}
L=\mu^{2}-\zeta(\lambda, \tau) \mu-\frac{1}{4}\left(\theta^{2}-1\right) \wp(\lambda, \tau), \\
H=\mu
\end{array}\right.
$$

(Assumptions (4) and (8) are simply that $\lambda \not \equiv 0 \bmod \Gamma_{\tau}$.) Consequently, applying Theorem 2 to this setting then yields the following Hamiltonian system:

$$
\left\{\begin{array}{l}
\frac{d \lambda}{d \tau}=\frac{1}{2 \pi \sqrt{-1}}[2 \mu-\varphi(\lambda, \tau)] \\
\frac{d \mu}{d \tau}=\frac{1}{2 \pi \sqrt{-1}}\left[\mu \varphi^{\prime}(\lambda, \tau)-\frac{1}{4}\left(\theta^{2}-1\right) \varphi^{\prime \prime}(\lambda, \tau)\right],
\end{array}\right.
$$

where we set $\varphi(\lambda, \tau)=\zeta(\lambda, \tau)-\eta_{1}(\tau) \lambda$, and denote by primes the differentiation with respect to $\lambda$.

To obtain the desired differential equation, we solve the first formula in (65) for $\mu$ and then substitute it into the second formula. In the course of the calculation, it should be remarked that the function $\varphi(\lambda, \tau)$ satisfies the equation

$$
\frac{\partial \varphi}{\partial \tau}(\lambda, \tau)=\frac{1}{2 \pi \sqrt{-1}}\left[\frac{1}{2} \varphi^{\prime \prime}(\lambda, \tau)+\varphi(\lambda, \tau) \varphi^{\prime}(\lambda, \tau)\right]
$$

which is shown by rewriting $\varphi(\lambda, \tau)$ as $\varphi(\lambda, \tau)=\theta_{1}^{\prime}(\lambda, \tau) / \theta_{1}(\lambda, \tau)$ via the odd theta function

$$
\theta_{1}(\lambda, \tau)=\sum_{n \in \mathbb{Z}} \exp \left[\pi \sqrt{-1} \tau\left(n+\frac{1}{2}\right)^{2}+2 \pi \sqrt{-1}\left(n+\frac{1}{2}\right)\left(\lambda+\frac{1}{2}\right)\right]
$$

and using the heat equation

$$
\theta_{1}^{\prime \prime}(\lambda, \tau)=4 \pi \sqrt{-1} \frac{\partial \theta_{1}}{\partial \tau}(\lambda, \tau)
$$

for $\theta_{1}(\lambda, \tau)$. As a result, we then obtain

$$
\frac{d^{2} \lambda}{d \tau^{2}}=-\frac{\theta^{2}}{8 \pi^{2}} \wp^{\prime}(\lambda, \tau)
$$

and thus complete the proof of Theorem 3. 


\section{REFERENCES}

[1] I. Biswas and K. Guruprasad, Principal bundles on open surfaces and invariant functions on Lie groups, Internat. J. Math., 4 (1993), 535-544.

[2] W. M. Goldman, The symplectic nature of fundamental groups of surfaces, Adv. Math., 54 (1984), 200-225.

[3] R. C. Gunning, Lectures on Riemann surfaces, Math. Notes, No. 2, Princeton Univ. Press, Princeton, NJ, 1966.

[4] L Lectures on vector bundles over Riemann surfaces, Math. Notes, No. 6, Princeton Univ. Press, Princeton, NJ, 1967.

[5] — Analytic structures on the space of flat vector bundles over a compact Riemann surface, Several complex variables, II (Proceedings of the International Conference, Univ. Maryland, College Park, MD, 1970), pp. 47-62, Lecture Notes in Math., Vol. 185, Springer-Verlag, Berlin, 1971.

[6] K. Iwasaki, Moduli and deformation for Fuchsian projective connections on a Riemann surface, J. Fac. Sci. Univ. Tokyo Sect. IA Math., 38 (1991), 431-531.

[7] —, Fuchsian moduli on a Riemann surface — its Poisson structure and Poincaré-Lefschetz duality, Pacific J. Math., 155 (1992), 319-340.

[8] K. Iwasaki, H. Kimura, S. Shimomura and M. Yoshida, From Gauss to Painlevé, Aspects Math., E16, Vieweg, Braunschweig, 1991.

[9] S. Kawai, Deformation of complex structures on a torus and monodromy preserving deformation, Thesis (1995), University of Tokyo.

[10] — The symplectic nature of the space of projective connections on Riemann surfaces, Math. Ann., 305 (1996), 161-182.

[11] Isomonodromic deformation of Fuchsian-type projective connections on elliptic curves, Analysis of discrete groups, II (Kyoto, 1996), Sūrikaisekikenkyūsho Kōkyūroku, 1022 (1997), 53-57.

[12] — A remark on Manin's description of the sixth Painlevé equation, in preparation.

[13] D. A. Korotkin and H. Samtleben, On the quantization of isomonodromic deformations on the torus, Internat. J. Modern Phys., A 12 (1997), 2013-2029, hep-th/9511087.

[14] A. M. Levin and M. A. Olshanetsky, Hierarchies of isomonodromic deformations and Hitchin systems, Moscow Seminar in Mathematical Physics, pp. 223-262, Amer. Math. Soc. Transl. Ser. 2, Vol. 191, Amer. Math. Soc., Providence, RI, 1999.

[15] Yu. I. Manin, Sixth Painlevé equation, universal elliptic curve, and mirror of $\mathbb{P}^{2}$, Geometry of differential equations, pp. 131-151, Amer. Math. Soc. Transl. Ser. 2, Vol. 186, Amer. Math. Soc., Providence, RI, 1998, alg-geom/9605010.

[16] K. Okamoto, On Fuchs's problem on a torus, I, Funkcial. Ekvac., 14 (1971), 137-152.

[17] The Hamiltonian structure derived from the holonomic deformation of the linear ordinary differential equations on an elliptic curve, Sci. Papers College Arts Sci. Univ. Tokyo, 37 (1987), 1-11.

[18] - On the holonomic deformation of linear ordinary differential equations on an elliptic curve, Kyushu J. Math., 49 (1995), 281-308. 
[19] K. Takasaki, Gaudin model, KZ equation, and isomonodromic problem on torus, Lett. Math. Phys., 44 (1998), 143-156, hep-th/9711058.

[20] Llliptic Calogero-Moser systems and isomonodromic deformations, J. Math. Phys., 40 (1999), 5787-5821, math.QA/9905101.

\author{
Department of Mathematics \\ Tokyo Institute of Technology \\ Oh-okayama \\ Meguro-ku \\ Tokyo, 152-8551 \\ Japan \\ kawai@math.titech.ac.jp
}

